\begin{tabular}{lc} 
Sharif University of Technology & Scientia Iranica \\
SCIENTIA & Transactions F: Nanotechnology \\
I RAN ICA & http://scientiairanica.sharif.edu \\
\hline
\end{tabular}

\title{
Numerical analysis of MHD mixed convection flow in a parallelogramic porous enclosure filled with nanofluid and in the presence of magnetic field induction
}

\author{
O. Ghaffarpasand ${ }^{a, b, *}$ and D. Fazeli ${ }^{a}$ \\ a. Department of Physics, University of Isfahan, Isfahan, Iran. \\ b. Environmental Research Institute, University of Isfahan, Isfahan, Iran. \\ Received 15 April 2016; received in revised form 21 October 2017; accepted 27 January 2018
}

\author{
KEYWORDS \\ Parallelogramic \\ enclosure; \\ MHD mixed \\ convection; \\ Porous media; \\ Nanofluid; \\ Heat transfer; \\ Magnetic potential.
}

\begin{abstract}
The present study numerically investigated the mixed convection flow of nanofluid in a lid-driven parallelogramic porous enclosure subjected to a magnetic field. The induced magnetic field was also considered in terms of the magnetic potential to solve the magnetohydrodynamic (MHD) flow and temperature equations. The DarcyBrinkman-Forchheimer model with the Boussinesq approximation was adopted, and the finite volume method based on SIMPLE algorithm was utilized to solve the governing equations with appropriate boundary conditions in an orthogonal computational domain. The governing equations in a non-orthogonal physical domain were transformed into a computational domain in an orthogonal co-ordinate by co-ordinate transformations. It was shown that the flow field and heat transfer were greatly sensible for the skew angle variation. Magnetic potential circulated through the parallelogramic porous enclosure with either a high magnetic Reynolds number or magnetic permeability of the nanofluid. Results also indicated that the influence of the external magnetic field on fluid characteristics and heat transfer manifested various fashions, mainly depending on the effective area of the parallelogramic enclosure. Besides, the variations of heat transfer rates while adding nanoparticles or applying magnetic field were affected to some extent by porous medium permeability and Richardson number.
\end{abstract}

(C) 2018 Sharif University of Technology. All rights reserved.

\section{Introduction}

The study of MHD mixed convection flow in porous enclosures has been met with great interest by researchers due to its numerous important applications such as optimization of solidification processes, waste nuclear processing, magnetohydrodynamic accelerators

\footnotetext{
*. Corresponding author. Tel.: +980 313-7934843; Fax: +98 0 313-7934800

E-mail address: o.ghaffarpasand@gmail.com (O. Ghaffarpasand)
}

doi: $10.24200 /$ sci. 2018.20034 and generators, and thermal insulation systems [1-4]. Magnetohydrodynamics usually involves the interaction of electrically conducting fluid and electromagnetic fields, whereby a magnetic field can be used to control fluid motion and heat transfer [5-7].

The addition of a minor volume fraction of high thermal conducting nano-scale particles $(<100 \mathrm{~nm})$ to the base fluid is an innovative technique for improving heat transfer of such thermal systems. Firstly, Choi [8] called the product nanofluid. Due to the size and minor volume fraction of suspended nanoparticles, nanofluids usually form very stable colloidal systems, which will prevent rapid settling and reduce clogging in the wall of heat transfer devices [9-11]. In recent years, the 
problem of MHD mixed convection of nanofluids in the porous media has received a great deal of interest due to its importance in numerous engineering applications such as microelectronics, microfluids, transportation, biomedical, etc. [12]. Considerable research works on this problem have been reported in the literature. For instance, Murthy et al. [13] investigated the effect of magnetic field on flow, heat and mass transfer characteristics in the free convection along a vertical plate immersed in the porous medium saturated by a thermally stratified nanofluid. Pekmen and TezerSezgin [14] utilized the Dual Reciprocity Boundary Element Method (DRBEM) to study steady free convection in special shape enclosures filled with a fluid saturated porous medium under the influence of a magnetic field. Results showed that the motion of the fluid was suppressed as the strength of the magnetic field increased. Fersadou et al. [9] numerically studied MHD mixed convection flow of nanofluid in a vertical rectangular duct. Results showed the heat transfer enhancement by using a porous medium, a nanofluid, and a magnetic field when assisting mixed convection.

Shermet et al. [15] conducted a numerical study to investigate free convection of nanofluid in a square porous cavity. They observed that the reduction of the thermal conductivity of the solid matrix reduced heat transfer inside the porous cavity. Mabood et al. [16] numerically analyzed the heat and mass transfer of MHD stagnation point flow of nanofluid in a porous medium. Their study considered the effects of thermal radiation, viscous dissipation, and chemical reaction. Numerical results indicated that increasing the volume fraction of nanoparticles led to the enhancement of convective heat transfer.

It should be noted that all of the studies above did not take the induced magnetic into consideration and they were restricted to small values of magnetic Reynolds number. The primary benefit of this hypothesis, which is known as the low-Rem approximation, is a significant reduction of the number of equations required to be solved [17]. The magnetic Reynolds number (Rem) represents the characteristics ratio between advection and magnetic diffusion. However, Rem deals with both of magnetic permeability and electrical conductivity of the fluid, and the probable interaction between the fluid velocity and external magnetic field results in an induced magnetic field inside the fluid. The induced magnetic field strongly affects the fluid flow and heat transfer characteristics. In addition, it can control the flow formation more accurately and have many applications in the experimental and theoretical studies of MHD flow due to its utilization in many industrial and technological phenomena [18].

Among the literature published on this subject, Singh et al. [19] numerically studied the free convection in an electrically conducting fluid in a vertical channel, where the induced magnetic field was included in the flow analyses. Later on, Costa et al. [20] investigated natural convection in a porous cavity under the influence of an induced magnetic field. As shown, the effect of the induced magnetic field is always a reduction of the natural convection taking place inside the enclosure. Kumar and Singh [21] considered the effect of an induced magnetic field on unsteady hydro-magnetic free convection flow. Results showed that the induced magnetic field slowed down the fluid flow. Akbar et al. $[22,23]$ examined the interaction of nanoparticle copper with the base fluid water in an asymmetric channel in the presence of an induced magnetic field. Pekmen \& Tezer-Sezgin [24] used DRBEM with Houbolt's timeintegration scheme to investigate mixed convection flow in a lid-driven square cavity filled with a porous medium under the influence of a magnetic field, where the induced magnetic field was not neglected. Results illustrated that the flow was slowed down by either a decrease in the permeability of porous media or an increase in the strength of external magnetic field. Seth \& Sarkar [25] numerically investigated the hydromagnetic natural convection flow in the presence of the induced magnetic field. Results showed that the external applied magnetic field tended to decrease the induced magnetic field. Kumar [26] analyzed the influence of the induced magnetic field on transient natural convection of the vertical cone. He inferred that the induced magnetic field slowed down the convection processes.

Most of the existing studies in the literature are mainly concentrated on rectangular or square enclosures. However, the heat transfer behavior in the parallelogramic porous and non-porous enclosures is more interesting, compared to the square and rectangular cavities, due to the presence of inclined walls. The parallelogramic enclosure is a geometry with interesting features and potentials in what concerns heat transfer performance. It has different heat transfer performances for different skew angles and can even present very different heat transfer behaviors for either positive or negative skew angles, being referred to as a heat transfer diode. It has a strong potential to be utilized as the basic structure which leads to a high-performance heat transfer element used in many various areas such as building applications, solar collectors, electronic thermal control geothermal applications, etc. [27-30]. The parallelogramic enclosure is a form that designates also a strong potential to a variable geometry structure, whereby the skew angle is changed by rotating the inclined walls of the enclosure to achieve the desired thermal performance each time. It also presents a special potential to act as a heat transfer inhibitor [31].

Among researchers investigating the convection in the parallelogramic enclosures, Al-Farhany and 
Turan [32] performed numerical simulations to study double diffusive natural convection inside an inclined rectangular cavity filled with a porous medium. Results showed that there was an inverse relation between the heat transfer and inclination angle. Chamkha at al. [33] numerically studied the natural convection of air in a differentially heated lid-driven parallelogramic enclosure. Their results indicate that Richardson number and inclination angle affect, to some extent, the convective current of heat transfer across the enclosure. Jagadeesha et al. [34] numerically investigated the double diffusive natural convection in an inclined parallelogramic porous enclosure. It was found that the inclination angle had significant effect on both of heat and mass transfer characteristics. Le Dez and Sadat [35] exhibited an exact analytical description of the internal radiative field inside the parallelogramic cavity.

Despite some available contents on natural convection in inclined enclosures, only a few research works have explored the natural convection of nanofluids in those geometries. Hussein \& Hussain [36] utilized numerical methods to study natural convection of copper-water nanofluid in a parallelogramic enclosure. Later, Nayak et al. [37] numerically studied the mixed convection of $\mathrm{Cu}$-water nanofluid in a skewed cavity. Results showed that the flow field was sensible, to some extent, for the skew angle variation, while heat transfer rate improved greatly by the addition of nanoparticles. All of the studies above were conducted in the absence of an external magnetic field. Recently, Ghaffarpasand [38] utilized an accurate finite volume method to study MHD mixed convection of nanofluids in a skewed porous cavity and in the presence of internal Joule heating and the absence of induced magnetic field. Results indicated that the permeability of porous media as well as the skew angle of skewed cavity influenced, to some extent, the heat transfer characteristics.

Based on a meticulous survey of the existing literature, less attention has been paid to convection of nanofluids in the presence of induced magnetic field. In particular, to the authors' best knowledge, the problem of MHD mixed convection inside a parallelogramic porous enclosure filled with nanofluid and in the presence of the induced magnetic field has not been analysed yet. Hence, the main objective of this work is to numerically examine MHD mixed convection and heat transfer of nanofluid inside a parallelogramic porous enclosure and exactly when the induced magnetic field is not neglected. For this purpose, the current paper studies the influence of permeability of the porous medium, magnetic permeability of nanofluid, external magnetic field, and Richardson number on the flow field, heat transfer, and magnetic potential of the flow in the various parallelogramic porous enclosures. In addition, it investigates the role of metallic nanoparticles in heat transfer enhancement in different flow configurations. To the authors' best knowledge, no such study has been reported to date in the literature.

\section{Problem definition and mathematical model}

The problem configuration, illustrated in Figure 1, is a parallelogramic porous enclosure whose bottom wall is along the $x$-axis, and sidewalls form angle $\lambda$ with $x$-axis. The left and right walls are assumed adiabatic, while the bottom wall of the enclosure is set at higher temperature $T_{h}$ as compared to top wall $T_{c}$. The top wall also moves with uniform velocity $U_{0}$ towards the right. Besides, the porous media of the cavity are saturated by $\mathrm{Cu}$-water nanofluid, whereby both the fluid phase and the nanoparticles are in thermal equilibrium and have the same velocity. The nanofluid is Newtonian, incompressible, and laminar; moreover, the porous medium is hydrodynamically, thermally, and electrically isotropic in the local thermal equilibrium with the nanofluid. The thermo-physical properties of the utilized nanofluid are given in Table 1. It is assumed that nanoparticles suspended in nanofluid are smaller than matrix pores, and agglomeration and deposition of those nanoparticles on the porous

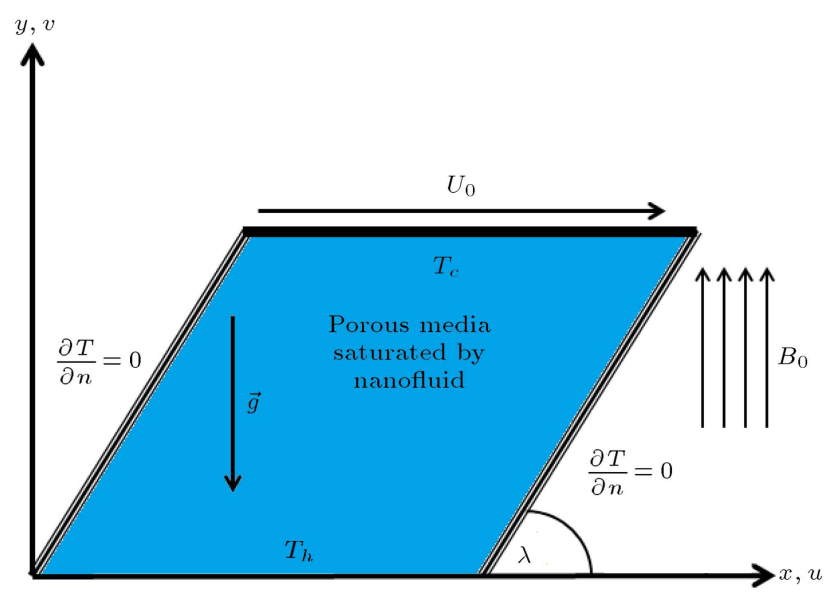

Figure 1. Problem configuration.

Table 1. Thermo-physical properties of pure water and copper nanoparticles [?].

\begin{tabular}{cccccc}
\hline $\begin{array}{c}\text { Physical } \\
\text { properties }\end{array}$ & $\begin{array}{c}\boldsymbol{\rho} \\
\left(\mathbf{k g m}^{-\mathbf{3}}\right)\end{array}$ & $\begin{array}{c}\boldsymbol{C}_{\boldsymbol{p}} \\
\left(\mathbf{J k g}^{-\mathbf{1}} \mathbf{K}^{-\mathbf{1}}\right)\end{array}$ & $\left.\begin{array}{c}\boldsymbol{k} \\
(\mathbf{W m}\end{array} \mathbf{W ~}^{-\mathbf{1}}\right)$ & $\begin{array}{c}\boldsymbol{\beta} \times \mathbf{1 0}^{-\mathbf{5}} \\
\left(\mathbf{K}^{-\mathbf{1}}\right)\end{array}$ & $\begin{array}{c}\boldsymbol{\sigma} \\
\left(\mathbf{S m}^{-\mathbf{1}}\right)\end{array}$ \\
\hline Water & 997.1 & 4179 & 0.613 & 21 & 0.05 \\
Copper & 8933 & 385 & 401 & 1.67 & $5.96 \times 10^{7}$ \\
\hline
\end{tabular}


matrix are negligible. The thermo-physical properties of the porous medium, base fluid, and nanoparticles are assumed to be constant except the density in the buoyancy term, which follows the Boussinesq approximation. Meanwhile, the radiated heat transfer and the chemical reaction between the base fluid and nanoparticles are neglected. The viscous dissipation, pressure work, joule heating, and Hall effect are all assumed negligible. An external magnetic field is applied in $+y$-direction. Besides, it is assumed that there is no any applied voltage implying the absence of an electric field.

The governing equations for this problem are based on the conservation of mass, momentum, and energy equation taking into account the presence of porous medium saturated by nanofluid, magnetic effect, and shear and buoyancy forces. Based on the above assumptions and by adopting nanofluid model proposed by Tiwari and Das [39] associated with the extended Brinkman-Forchheimer Darcy model to incorporate the viscous and inertia effects, the equations are given in the vector form as follows [9]:

$$
\begin{aligned}
& \nabla \cdot \mathbf{u}=0 \\
& \frac{\mu_{e f f}}{\varepsilon} \nabla^{2} \mathbf{u}=\frac{\rho_{n f}}{\varepsilon^{2}}\left[\frac{\partial \mathbf{u}}{\partial t}+(\mathbf{u} \cdot \nabla) \mathbf{u}\right]+\nabla p+\frac{\mu_{n f}}{K} \mathbf{u} \\
& +\rho_{n f} \frac{C}{\sqrt{K}}|\mathbf{u}| \mathbf{u}+(\rho \beta)_{n f}\left(T-T_{c}\right) \mathbf{g} \\
& -\mathbf{J} \times \mathbf{B}, \\
& k_{e f f} \nabla^{2} T=\left(\rho C_{P}\right)_{n f}\left[(\mathbf{u} \cdot \nabla) T+\frac{\partial T}{\partial t}\right] .
\end{aligned}
$$

It should be noted that the vectors are written in bold. The current density vector $\mathbf{J}$ is related to electrical and magnetic fields by the Ohm's law as follows:

$$
\mathbf{J}=\sigma_{n f}(\mathbf{E}+\mathbf{u} \times \mathbf{B}),
$$

where $\sigma_{n f}$ is the electrical conductivity of the nanofluid. The low-frequency Ampere's law neglects the displacement current and is given by $\mu_{0} \mathbf{J}=\nabla \times \mathbf{B}$. By neglecting displacement current, using solenoidal nature of $\mathbf{B}$, and Faraday's law $\nabla \times \mathbf{E}=-\frac{\partial \mathbf{B}}{\partial t}$, the magnetic induction equation can be derived as follows:

$$
\frac{1}{\sigma_{n f} \mu_{m}} \nabla^{2} \mathbf{B}=\frac{\partial \mathbf{B}}{\partial t}-\nabla \times(\mathbf{u} \times \mathbf{B}) .
$$

Magnetic induction relates to magnetic potential $(\mathbf{B}=$ $\nabla \times \mathbf{A})$, whereby the above equation can be reduced to the same equation for the magnetic potential as follows:

$$
\frac{1}{\sigma_{n f} \mu_{m}} \nabla^{2} A=\frac{\partial A}{\partial t}+u \frac{\partial A}{\partial x}+v \frac{\partial A}{\partial y},
$$

where $\mu_{m}$ is the magnetic permeability. Therefore, the main governing equations in the non-dimensional form can be expressed as follows:

$$
\begin{aligned}
& \frac{\partial u}{\partial x}+\frac{\partial v}{\partial y}=0 \\
& \frac{\partial u}{\partial \tau}+\frac{\partial(u u)}{\partial x}+\frac{\partial(v u)}{\partial y}=-\frac{\partial P}{\partial x} \\
& +\frac{\rho_{f}}{\rho_{n f}} \frac{\mu_{n f}}{\mu_{f}} \frac{R_{\mu}}{\operatorname{Re}}\left(\frac{\partial^{2} u}{\partial x^{2}}+\frac{\partial^{2} u}{\partial y^{2}}\right)-\frac{\rho_{f}}{\rho_{n f}} \frac{\mu_{n f}}{\mu_{f}} \frac{\varepsilon}{\operatorname{ReDa}} u \\
& -\frac{\varepsilon^{2} C}{\sqrt{\mathrm{Da}}}|\mathbf{u}| u+\frac{\rho_{f}}{\rho_{n f}} \frac{\varepsilon H a^{2}}{\operatorname{ReRem}} B_{y}\left(\frac{\partial B_{y}}{\partial x}-\frac{\partial B_{x}}{\partial y}\right), \\
& \frac{\partial v}{\partial \tau}+\frac{\partial(u v)}{\partial x}+\frac{\partial(v v)}{\partial y}=-\frac{\partial P}{\partial y} \\
& +\frac{\rho_{f}}{\rho_{n f}} \frac{\mu_{n f}}{\mu_{f}} \frac{R_{\mu}}{\operatorname{Re}}\left(\frac{\partial^{2} v}{\partial x^{2}}+\frac{\partial^{2} v}{\partial y^{2}}\right)-\frac{\rho_{f}}{\rho_{n f}} \frac{\mu_{n f}}{\mu_{f}} \frac{\varepsilon}{\operatorname{Re~Da} v} v \\
& -\frac{\varepsilon^{2} C}{\sqrt{\mathrm{Da}}}|\mathbf{u}| v+\frac{\rho_{f}}{\rho_{n f}} \frac{(\rho \beta)_{n f}}{(\rho \beta)_{f}} \frac{\rho_{f}}{\rho_{n f}} \operatorname{Ri} \Theta \\
& +\frac{\rho_{f}}{\rho_{n f}} \frac{\varepsilon H a^{2}}{\operatorname{ReRem}} B_{x}\left(\frac{\partial B_{y}}{\partial x}-\frac{\partial B_{x}}{\partial y}\right), \\
& \frac{\partial \Theta}{\partial \tau}+\frac{\partial(u \Theta)}{\partial x}+\frac{\partial(v \Theta)}{\partial y} \\
& =\frac{R_{k}}{\varepsilon \operatorname{Re} \operatorname{Pr}} \frac{k_{n f}}{k_{f}} \frac{\left(\rho C_{P}\right)_{f}}{\left(\rho C_{P}\right)_{n f}}\left(\frac{\partial^{2} \Theta}{\partial x^{2}}+\frac{\partial^{2} \Theta}{\partial y^{2}}\right), \\
& \frac{\partial A}{\partial \tau}+\frac{\partial(u A)}{\partial x}+\frac{\partial(v A)}{\partial y}=\frac{1}{\varepsilon \operatorname{Rem}}\left(\frac{\partial^{2} A}{\partial x^{2}}+\frac{\partial^{2} A}{\partial y^{2}}\right)
\end{aligned}
$$

where $B_{x}=\frac{\partial A}{\partial y}$, and $B_{y}=-\frac{\partial A}{\partial x}$. The following variables are used for non-dimensionalizing the governing equations [38]:

$$
\begin{aligned}
& (x, y)=\frac{\left(x^{\prime}, y^{\prime}\right)}{L}, \quad(u, v)=\frac{\left(u^{\prime}, v^{\prime}\right)}{\varepsilon U_{0}}, \quad \Theta=\frac{T-T_{c}}{T_{h}-T_{c}}, \\
& A=\frac{A^{\prime}}{B_{0} L}, \quad \tau=\frac{\varepsilon t U_{0}}{L}, \quad P=\frac{p}{\rho_{n f} U_{0}^{2}} .
\end{aligned}
$$

Superscript ' is utilized for dimensional parameters. The effective density at the reference temperature, thermal diffusivity, heat capacitance, and thermal expansion coefficient of the nanofluids based on classical models can be written as follows [22]:

$$
\begin{aligned}
& \rho_{n f}=(1-\phi) \rho_{f}+\phi \rho_{s}, \\
& \alpha_{n f}=\frac{k_{n f}}{\left(\rho C_{p}\right)_{n f}},
\end{aligned}
$$




$$
\begin{aligned}
& \left(\rho C_{p}\right)_{n f}=(1-\phi)\left(\rho C_{p}\right)_{f}+\phi\left(\rho C_{P}\right)_{s}, \\
& (\rho \beta)_{n f}=(1-\phi)(\rho \beta)_{f}+\phi(\rho \beta)_{s},
\end{aligned}
$$

where $\phi$ is the solid volume fraction of the nanoparticles. The effective thermal conductivity of nanofluid is approximated by the Maxwell self-consistent approximation model, whereby, for the two-component entity of spherical-particle suspension, $k_{n f}$ can be expressed as follows $[22,40]$ :

$$
\frac{k_{n f}}{k_{f}}=\frac{k_{s}+2 k_{f}-2 \phi\left(k_{f}-k_{s}\right)}{k_{s}+2 k_{f}+\phi\left(k_{f}-k_{s}\right)} .
$$

The viscosity of the nanofluid is calculated using the Brinkman model as follows:

$$
\mu_{n f}=\frac{\mu_{f}}{(1-\phi)^{2.5}} .
$$

The electrical conductivity of the nanofluid is calculated by Maxwell model as follows:

$$
\frac{\sigma_{n f}}{\sigma_{f}}=1+\frac{3(\xi-1) \phi}{(\xi+2)-(\xi-1) \phi},
$$

where $\xi=\frac{\sigma_{s}}{\sigma_{f}}$. The boundary conditions associated with the problem in physical domain are as follows:

$u=1, \quad v=0, \quad \Theta=0$

for $y=\sin \lambda$, and $\cos \lambda \leq x \leq \cos \lambda+1 \quad$ (Top wall)

$u=v=0, \quad \Theta=1$

for $y=0$, and $0 \leq x \leq 1 \quad$ (Bottom wall)

$u=v=0, \quad \frac{\partial \Theta}{\partial n}=0$,

for $x=y \cos \lambda(0 \leq y \leq 1), \quad 0 \leq y \leq \sin \lambda$ (Left wall)

$u=v=0, \quad \frac{\partial \Theta}{\partial n}=0$,

for $\quad x=1+y \cos \lambda(0 \leq y \leq 1) 0 \leq y \leq \sin \lambda$ (Right wall).

Herein, $n$ is the normal displacement with respect to the left and right sided walls. Related boundary conditions are allowed to take zero stream function on the walls. However, the external magnetic field is applied in the $+y$-direction so that $A=-x+C^{*}$ on the boundaries, where $C^{*}$ is a constant. Constant $C^{*}$ is assumed zero with a similar idea in obtaining constant stream function values as zero on the walls. Therefore, $A=-x$ at $y=0,1$, and $A=-y \cos \lambda$ and $-1-y \cos \lambda$ at the left and right walls, respectively, where $0 \leq y \leq 1$. The problem of MHD convection is characterized herein by the following dimensionless parameters:

$$
\begin{aligned}
& \mathrm{Ri}=\frac{\mathrm{Gr}}{\operatorname{Re}^{2}}=\frac{g \beta \Delta T L^{3} / \nu_{f}^{2}}{U_{0} L / \nu_{f}}, \quad \operatorname{Pr}=\frac{\nu_{f}}{\alpha_{f}}, \\
& \mathrm{Ha}=B_{0} \sqrt{\frac{\sigma_{f} L}{\mu_{f}}}, \quad \operatorname{Rem}=\mu_{m} \sigma_{n f} U_{0} L, \\
& R_{\mu}=\frac{\mu_{e f f}}{\mu_{n f}}, \quad R_{k}=\frac{k_{e f f}}{k_{n f}}
\end{aligned}
$$

where Ri, Gr, Re, Rem, Pr, and Ha are Richardson, Grashof, Reynolds, magnetic Reynolds, Prandtl, and Hartman numbers, respectively, and $R_{\mu}$ and $R_{k}$ are the thermal conductivity and viscous ratio porousnanofluid, respectively. In this study, a co-ordinate transformation is utilized to transform physical domain in $x, y$ plane into an orthogonal system in the computational domain. Nayak et al. [37] introduced this method before. For this purpose, independent variables, $x, y$, in the physical domain are transformed into the independent variables, $\zeta, \eta$, in the computational domain by the following relations:

$$
\zeta=x-y \cot \lambda, \quad \eta=y / \sin \lambda .
$$

Under this transformation, the non-dimensional transformed governing equations in the orthogonal computational domain can be written as follows:

$$
\begin{aligned}
& \frac{\partial}{\partial \zeta}(u-v \cot \lambda)+\frac{\partial}{\partial \eta}\left(\frac{v}{\sin \lambda}\right)=0, \\
& \frac{\partial u}{\partial \tau}+\frac{\partial}{\partial \zeta} u(u-v \cot \lambda)+\frac{\partial}{\partial \eta} u\left(\frac{v}{\sin \lambda}\right)=-\frac{\partial P}{\partial \zeta} \\
& \quad+\frac{R_{\mu}}{\operatorname{Re}} \frac{\mu_{n f}}{\mu_{f}} \frac{\rho_{f}}{\rho_{n f}} a\left(\nabla_{\zeta \eta}^{2} u\right)-\frac{\varepsilon^{2} C}{\sqrt{\mathrm{Da}}}|\mathbf{u}| u \\
& \quad-\frac{\mu_{n f}}{\mu_{f}} \frac{\rho_{f}}{\rho_{n f}} \frac{\varepsilon}{\operatorname{ReDa}} u+\frac{\rho_{f}}{\rho_{n f}} \frac{\varepsilon \mathrm{Ha}^{2}}{\operatorname{Re} \operatorname{Rem}_{\eta}}\left[B_{\eta}\left(-\nabla_{\zeta \eta}^{2} A\right)\right],
\end{aligned}
$$

$$
\begin{aligned}
\frac{\partial v}{\partial \tau} & +\frac{\partial}{\partial \zeta} v(u-v \cot \lambda)+\frac{\partial}{\partial \eta} v\left(\frac{v}{\sin \lambda}\right)= \\
& +\cot \lambda \frac{\partial P}{\partial \zeta}+\left(1-\frac{1}{\sin \lambda}\right) \frac{\partial P}{\partial \eta} \\
& +\frac{R_{\mu}}{\operatorname{Re}} \frac{\mu_{n f}}{\mu_{f}} \frac{\rho_{f}}{\rho_{n f}} a\left(\nabla_{\zeta \eta}^{2} v\right)-\frac{\varepsilon^{2} C}{\sqrt{\mathrm{Da}}}|\mathbf{u}| v \\
& -\frac{\mu_{n f}}{\mu_{f}} \frac{\rho_{f}}{\rho_{n f}} \frac{\varepsilon}{\operatorname{ReDa}} v+\frac{\rho_{f}}{\rho_{n f}} \frac{(\rho \beta)_{n f}}{(\rho \beta)_{f}} \operatorname{Ri\Theta } \\
& -\frac{\rho_{f}}{\rho_{n f}} \frac{\varepsilon \mathrm{Ha}^{2}}{\operatorname{Re} \operatorname{Rem}}\left[B_{\zeta}\left(-\nabla_{\zeta \eta}^{2} A\right)\right],
\end{aligned}
$$




$$
\begin{array}{r}
\frac{\partial \Theta}{\partial \tau}+\frac{\partial}{\partial \zeta} \Theta(u-v \cot \lambda)+\frac{\partial}{\partial \eta} \Theta\left(\frac{v}{\sin \lambda}\right) \\
=\frac{R_{k}}{\varepsilon \operatorname{Re} \operatorname{Pr}} \frac{k_{n f}}{k_{f}} \frac{\left(\rho C_{p}\right)_{f}}{\left(\rho C_{p}\right)_{n f}} a\left(\nabla_{\zeta \eta}^{2} \Theta\right), \\
\frac{\partial A}{\partial \tau}+\frac{\partial}{\partial \zeta} A(u-v \cot \lambda)+\frac{\partial}{\partial \eta} A\left(\frac{v}{\sin \lambda}\right) \\
=\frac{1}{\varepsilon \operatorname{Rem}} a\left(\nabla_{\zeta \eta}^{2} A\right) .
\end{array}
$$

Here:

$$
\begin{aligned}
& B_{\zeta}=-\cot \lambda \frac{\partial A}{\partial \zeta}+\frac{1}{\sin \lambda} \frac{\partial A}{\partial \eta}, \\
& B_{\eta}=-\frac{\partial A}{\partial \eta}, \\
& \nabla_{\zeta \eta}^{2}=a\left(\frac{\partial^{2}}{\partial \zeta^{2}}+\frac{\partial^{2}}{\partial \eta^{2}}-2 c \frac{\partial^{2}}{\partial \zeta \partial \eta}\right),
\end{aligned}
$$

where $a=\operatorname{cosec}^{2} \lambda$, and $c=\cos \lambda$. The boundary conditions in the computational domain are:

$$
\begin{aligned}
& u=1, \quad v=0, \quad \Theta=0, \quad A=-\zeta, \quad \text { at } \quad \eta=1, \\
& u=v=0, \quad \Theta=1, \quad A=-\zeta, \quad \text { at } \quad \eta=0, \\
& u=v=0, \quad \frac{\partial \Theta}{\partial \zeta}=\cos \lambda \frac{\partial \Theta}{\partial \eta}, \quad A=-\eta \cos \lambda, \\
& \text { at } \quad \zeta=0, \\
& u=v=0, \quad \frac{\partial \Theta}{\partial \zeta}=\cos \lambda \frac{\partial \Theta}{\partial \eta}, \quad A=-1-\eta \cos \lambda, \\
& \text { at } \zeta=1 .
\end{aligned}
$$

It should be noted that the governing equations are solved in an orthogonal computational domain by a method explained in detail in the next section. Average Nusselt number $\overline{\mathrm{Nu}}$ on the bottom heated wall is investigated to examine heat transfer across the parallelogramic enclosure, whereby the local and average Nusselt numbers are calculated as follows:

$$
\mathrm{Nu}=-\frac{k_{n f}}{k_{f}} \frac{\partial \Theta}{\partial \eta}, \quad \overline{\mathrm{Nu}}=\int_{0}^{1} \mathrm{Nu} d \zeta
$$

In order to quantify the effect of nanoparticles volume fraction and the external magnetic field on convective heat transfer, the following average Nusselt numbers are also defined and calculated:

$$
\overline{\mathrm{Nu}}^{*}=\frac{\overline{\mathrm{Nu}}(\phi)}{\overline{\mathrm{Nu}}(\phi=0)} \quad \overline{\mathrm{Nu}}^{* *}=\frac{\overline{\mathrm{Nu}}(\mathrm{Ha})}{\overline{\mathrm{Nu}}(\mathrm{Ha}=0)} .
$$

\section{Numerical solution}

The transformed Navier-Stokes and energy equations are numerically solved using the control volume approach in a staggered grid arrangement. A secondorder upwind scheme is used for the discretization of the convective terms, and a second-order central difference is used for the discretization of the diffusion terms. The SIMPLE algorithm is then implemented for pressure and velocity coupling. During the SIMPLE iteration process, the pressure correction method is established to obtain the real velocity field. The set of resulting algebraic equations is solved iteratively and is performed by a coupling manner through the block elimination algorithm. Considering the convergence of the numerical results, the under-relaxation method is utilized, whereby the following criterion is adopted to secure steady conditions:

$$
\sum_{i, j}\left|\Phi_{i, j}^{m+1}-\Phi_{i, j}^{m}\right| \leq 10^{-6}
$$

where generic variable $\Phi$ represents the set of four variables, $u, v, \mathrm{~A}$, or $\Theta$. In the above inequality, superscripts $m$ indicate the iteration index, and the subscript sequence $(i, j)$ denotes the iteration index. The grid independence study is also carried out for the three different grids, namely $90 \times 90,125 \times 125$, and $150 \times 150$ for a differentially heated parallelogramic enclosure with a skew angle $\lambda=45^{\circ}$ at $\mathrm{Ri}=10, \phi=0.05$, and $\mathrm{Ha}=25$. Considering simulated accuracy and CPU time in the range of variables, the uniform grid of $125 \times 125$ is found sufficiently fine to ensure the grid independent solution and is utilized for all subsequent simulations. The influence of Darcy number, magnetic Reynolds number, Hartman number, and Richardson number is analyzed and discussed through this study. All computations are carried out for a time step close to $\Delta \tau=0.25$. Only for cases with $\mathrm{Ha}=50$ and 100 , a relaxation parameter $0<\gamma<1$ is utilized for convergence of velocity components where $(u, v)^{m+1} \rightarrow$ $\gamma(u, v)^{m+1}+(1-\gamma)(u, v)^{m}$. The used $\gamma$ and $\Delta \tau$ for cases with $\mathrm{Ha}=50$ and 100 are $\gamma=0.5, \Delta \tau=0.2$, and $\gamma=0.1, \Delta \tau=0.1$, respectively. It is also worthwhile to note that all simulation results are reported in the steady-state conditions.

To ensure the credibility of the numerical method, the developed code is validated based on some former published results in the literature. The case of liddriven skewed cavity flow due to pure fluid $(\phi=$ $0.0)$ is tested first. Considering that vertical velocity along the horizontal central line is obtained by the method developed in this study, findings of Demirdciz et al. [27] and Nayak et al. [37] are compared in Figure 2(a). As one can remark, excellent agreement is observed. A second test for the local Nusselt number along the hot wall for a lid-driven square cavity filled 


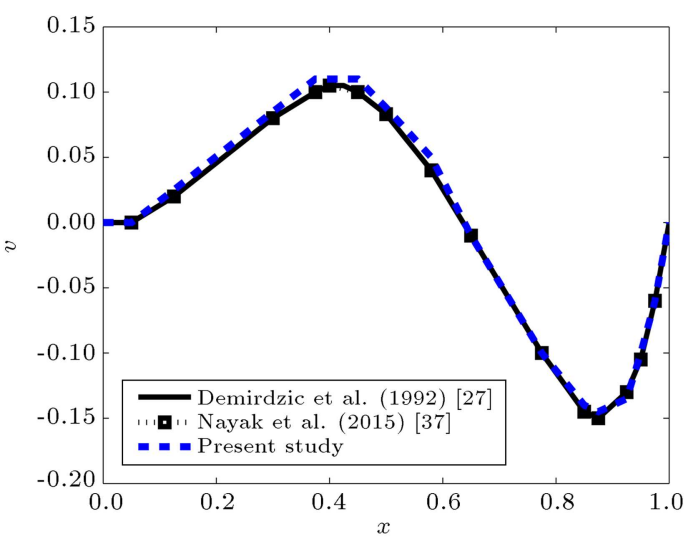

(a)

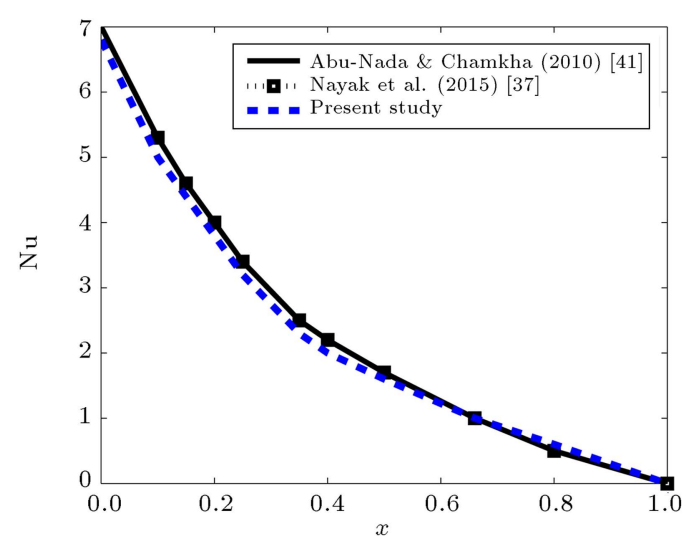

(b)

Figure 2. (a) The comparison of the present results for $v$-velocity profile with the numerical results due to Demirdzic et al. [27] and Nayak et al. [37], when $\operatorname{Re}=100$, and $\lambda=45^{\circ}$ for pure fluid $(\phi=0)$. (b) The comparison of the local Nusselt number along the hot wall with former results of Abu-Nada and Chamkha [41], and Nayak et al. [37], when Ri $=1$, $\lambda=90^{\circ}$, and $\phi=10 \%$.

with nanofluid $\phi=10 \%, \mathrm{Ri}=1$, and $\mathrm{Re}=100$ has been conducted. As shown in Figure 2(b), the computed Nusselt numbers exhibit good agreement with findings of Abu-Nada and Chamkha [41] and Nayak et al. [37]. These favorable comparisons lend confidence and validity to the numerical results, to be reported subsequently.

\section{Results and discussions}

A numerical investigation is carried out to study MHD mixed convection within a differentially heated parallelogramic porous lid-driven enclosure saturated by nanofluid for a wide range of key parameters such as skew angle $(\lambda)$, nanoparticle volume fraction $(\phi)$, Darcy number (Da), magnetic Reynolds number (Rem), Hartman number (Ha), and Richardson number $(\mathrm{Ri})$. Due to the great number of control parameters, all computations are performed by keeping fixed the enclosure length $(L=1)$, the type of nanofluid (copper-water), the Reynolds number ( $\operatorname{Re}=100)$, the Prandtl number of the base fluid $(\operatorname{Pr}=6.8)$, the porosity $(\varepsilon=0.9)$, the inertial coefficient $(C=$ $0.1)$, the viscosity ratio $\left(R_{\mu}=1\right)$, and the thermal conductivity ratio $\left(R_{k}=1\right)$. A similar trend has been carried out before in the study of Fersadou et al. [9]. The range of skew angle, solid volume fraction, Darcy number, magnetic Reynolds number, Hartman number, and Richardson number varies between $30^{\circ} \leq$ $\lambda \leq 150^{\circ}, 0 \leq \phi \leq 0.2,10^{-4} \leq \mathrm{Da} \leq 1$, $1 \leq \operatorname{Rem} \leq 100,0 \leq \mathrm{Ha} \leq 100$, and $0.01 \leq$ $\mathrm{Ri} \leq 10$, respectively. The induced magnetic field is considered in this study and analyzed in terms of the magnetic potential. The flow, thermal and magnetic potential fields are analyzed through the streamline, isotherm, and isopotential contours, respectively, and are presented just in parallelogramic enclosures with $\lambda=30^{\circ}, 90^{\circ}$, and $120^{\circ}$ for the sake of visibility. The heat transfer is characterized by calculating the average Nusselt number at the heated bottom wall. The variation of the absolute value of stream function at the centre of the primary vortex $\left|\psi_{\text {Max }}\right|$ is also studied to examine the influence of key parameters on the fluid intensity.

\subsection{Effect of porous medium permeability (Darcy number, Da)}

The effect of porous medium permeability on the fluid characteristics and heat transfer phenomena is conducted by analyzing wide cases with different Darcy numbers. For this purpose, the streamlines, isotherms, and magnetic isopotentials for $\mathrm{Ha}=5, \mathrm{Ri}=0.01$, $\mathrm{Da}=10^{-4}, 10^{-3}, 10^{-2}$, and 1 , and two values of solid volume fractions, i.e. solid lines for nanofluid $(\phi=6 \%)$ and dotted lines for pure fluid $(\phi=0)$, are illustrated in Figure 3(a), (b), and (c), respectively. It is worthwhile to note that the magnetic induction effect is also taken into account here, whereby Rem $=100$. Due to the thermal buoyancy and the imposed temperature gradient between the horizontal walls, the hot fluid rises up from the heated bottom wall and the cold fluid goes down along the cold cross wall. This feature leads to the formation of a clockwise unicellular flow pattern which occupies the almost whole enclosure as can be observed in Figure 3(a). According to the Richardson number value $(\mathrm{Ri}=0.01)$, the flow is dominated here by forced convection provoked by the lid's movement. Hence, shear forces affect the flow field greatly due to which the streamlines stretch rightwards near the moving top lid. However, shear forces tend to push the flow towards the bottom half of the cavity. With decreasing Darcy number, the effect of shear forces is diminished and the center of the streamlines clusters through the top wall. This fact resembles the 


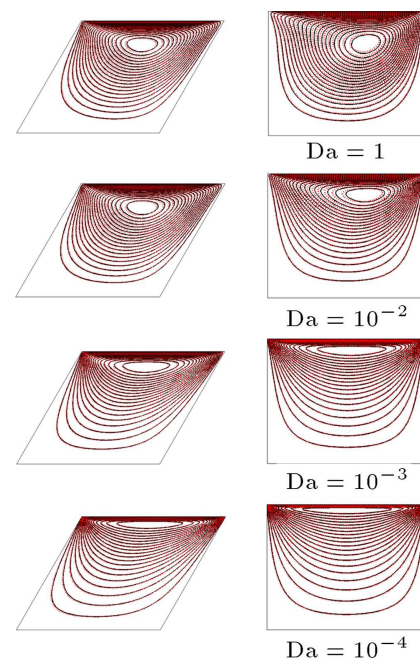

(a)
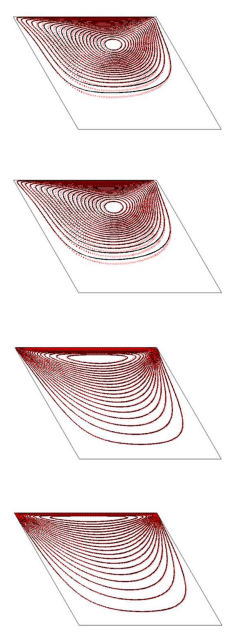
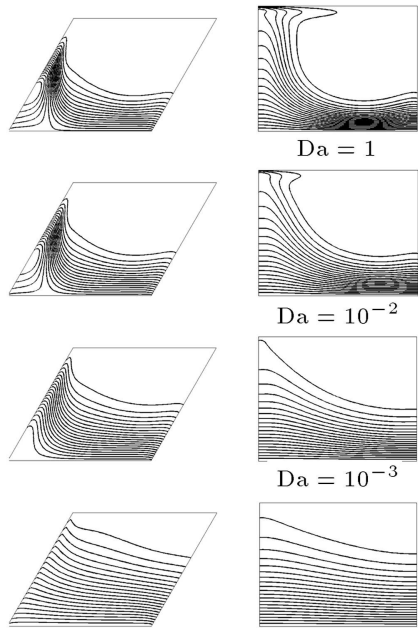

$\mathrm{Da}=10^{-4}$

(b)
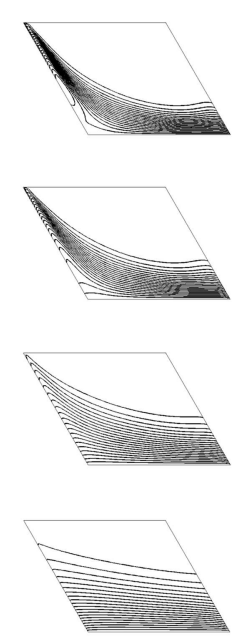
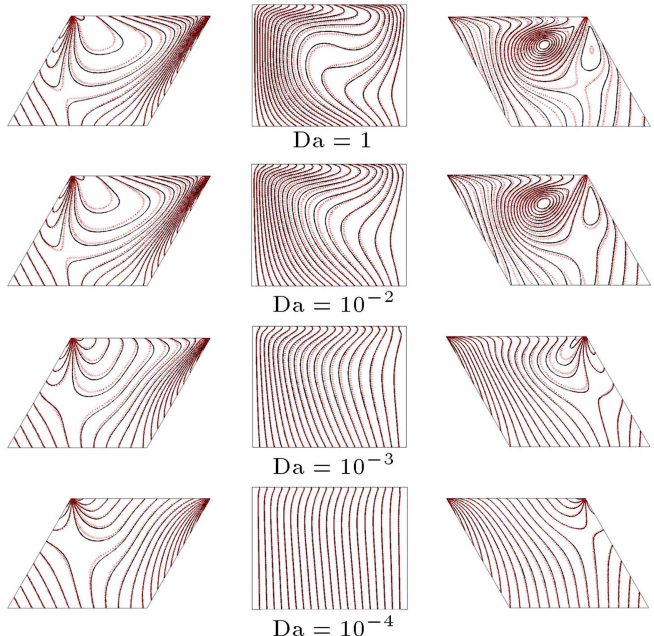

(c)

Figure 3. (a) Streamlines, (b) isotherms, and (c) magnetic isopotentials of MHD forced convection $(\mathrm{Re}=\mathrm{Gr}=100$, $\mathrm{Ri}=0.01$ ) of nanofluid in the parallelogramic porous enclosures with $\lambda=60^{\circ}$ (first column), $\lambda=90^{\circ}$ (second column), and $\lambda=120^{\circ}$ (third column), when Rem $=100$ and $\mathrm{Ha}=5$. Dotted lines are for the pure fluid $(\phi=0)$ and solid lines are for the nanofluid $(\phi=6 \%)$.

results of Pekmen and Tezer-Sezgin [24] for MHD flow of pure air $(\operatorname{Pr}=1)$ in a lid-driven square porous enclosure.

It is well known that the porous medium with small Darcy number has a small permeability. Hence, the fluid flow through the porous medium with small Darcy number faces high flow resistance within the porous bed. In other words, the fluid resistance against fluid flow in porous media is increased with decreasing Darcy number. This is the main reason for clustering the primary vortex near the top wall in cases with small Darcy numbers. On the other hand, it can be seen that the nanofluid flow inside a square porous cavity $\left(\lambda=90^{\circ}\right)$ has the largest primary vortex with respect to the nanofluid flow inside the other parallelogramic porous enclosures. This fact attributes to the effective area of the parallelogramic enclosures. As the skew angle of parallelogramic enclosures increases to $90^{\circ}$, the effective area enhances its maximum value, whereas it is reduced with further increasing of $\lambda$. The convection current of heat is developed greatly in parallelogramic enclosures with larger effective areas; therefore, nanofluid flow in the square enclosure has a larger primary vortex with respect to the flow in the other parallelogramic enclosures.

The effect of Darcy number on the temperature distribution, isotherm lines, of MHD flow of nanofluid in parallelogramic porous enclosures is illustrated in Figure 3(b). With a decrease of Darcy number, the concentration of isotherm lines near the heated wall is reduced, and they are nearly perpendicular to the sided walls. This feature is brought about by the fluid friction within the porous enclosure. The fluid resistance against convective current of heat across 
the enclosure is increased by reducing Darcy number, whereby the parallelogramic porous enclosure becomes a quasi-conductive domain as Darcy number decreases. The influence of Darcy number on magnetic isopotential contours is depicted in Figure 3(c). It can be seen that the distortions observed in isopotential contours are reduced with decreasing Darcy number. This fact represents that the diffusion and advection effects of magnetic field on isopotential contours increase and decrease with a further reduction of porous medium permeability, respectively. Figure 3(c) also shows that the influence of Darcy number on magnetic isopotential contours is augmented by adding metallic nanoparticles, whereby nanofluids (solid lines) have almost larger distortions with respect to the pure fluids (dotted lines).

The variation of absolute value of stream function at primary vortex $\left|\psi_{\mathrm{Max}}\right|$, as a function of skew angle, is illustrated in Figure 4(a) for various Darcy numbers. First, it can be seen that the value of $\left|\psi_{\mathrm{Max}}\right|$ reduces with increasing $\lambda$ from $30^{\circ}$ to $90^{\circ}$ and, then, increases with a further increase of the skew angle. In other words, the cases with $\lambda=90^{\circ}$ have the smallest $\left|\psi_{\text {Max }}\right|$ values. The square parallelogramic cavities have the largest effective areas and, consequently, deal with the largest fluid resistance within the porous bed. Hence, the fluid flow in those enclosures faces the largest fluid resistance and, consequently, has the smallest stream functions with respect to the other parallelogramic enclosures. The variation of the average Nusselt number as a function of the skew angle for various Darcy numbers is depicted in Figure 4(b). It can be seen that $\overline{\mathrm{Nu}}$ profile is almost symmetric around $\lambda=90^{\circ}$. The average Nusselt number is increased with the enhancement of the skew angle for $\lambda \leq 90^{\circ}$, and, then, it is reduced with a further increase of $\lambda$ values. This result which resembles the result of Nayak et al. [37] and Hussein and Hussain [36] for mixed convection of nanofluid in a skewed lid-driven enclosure is due to the effective area of the porous parallelogramic cavity. With the increase of skew angle from $30^{\circ}$ to $90^{\circ}$, the effective area of the parallelogramic porous enclosure and, further, the convection heat transfer are increased, while the inverse occurs with a further increase of $\lambda$ value.

However, the cases with lower Darcy numbers have smaller $\overline{\mathrm{Nu}}$ and $\left|\psi_{\text {Max }}\right|$ values. Increasing the flow resistance in porous media with lower and moder-

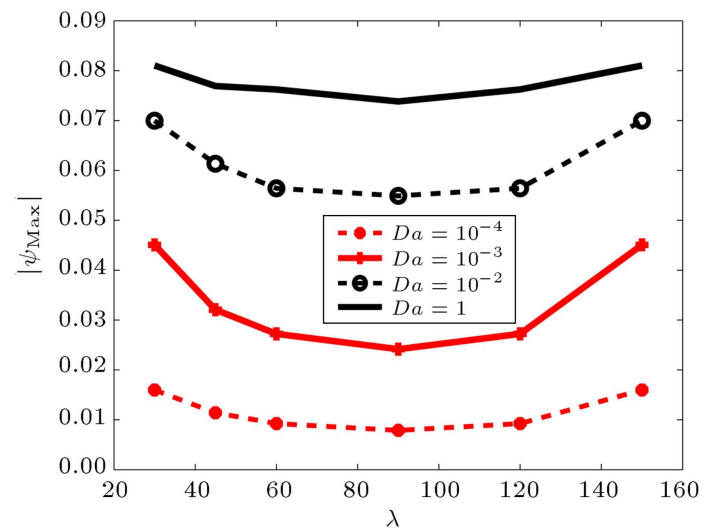

(a)

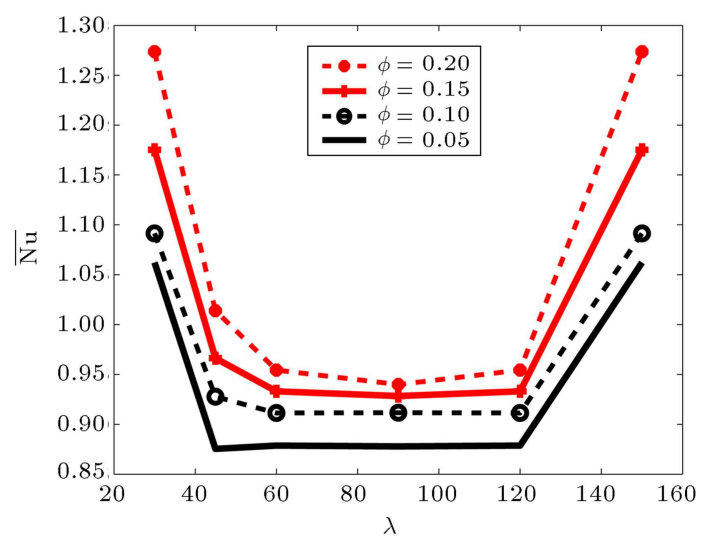

(c)

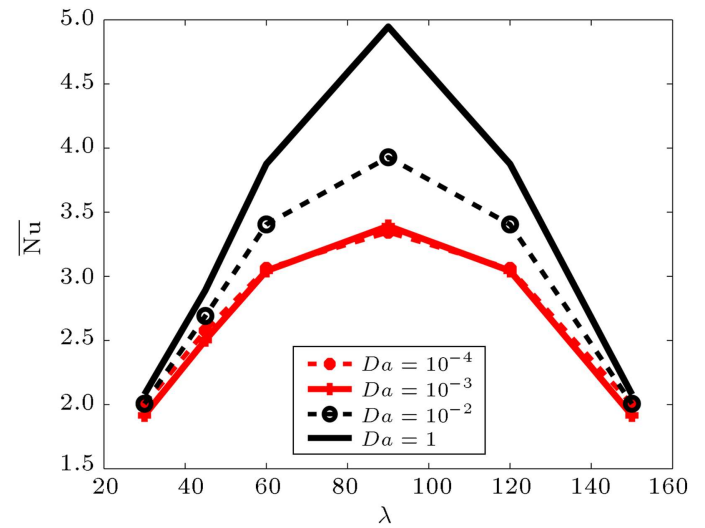

(a)

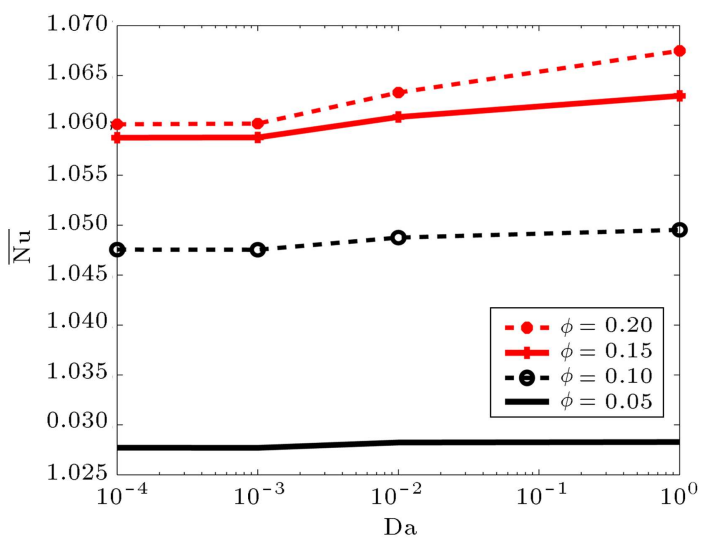

(d)

Figure 4. The variation of (a) absolute value of stream function of primary vortex and (b) average Nusselt number as a function of skew angle for different Darcy numbers. The variation of $\overline{\mathrm{Nu}}^{*}$ as a function of (c) skew angle and (d) Darcy number for various solid volume fractions. In all of figures $\mathrm{Ha}=5, \mathrm{Rem}=100, \mathrm{Re}=\mathrm{Gr}=100$, and $\mathrm{Ri}=0.01$. 
ate permeabilities reduces greatly the convective heat transfer and fluid velocity in parallelogramic porous enclosure; thus, the cases with larger Darcy numbers have larger $\left|\psi_{\text {Max }}\right|$ and $\overline{\mathrm{Nu}}$ values. The reduction effect of Darcy number against convective heat transfer has been also observed before by Shermet et al. [3] and Fersadou et al. [9] in rectangular and square enclosures. The variations in rates of the average Nusselt number as a function of the skew angle and Darcy number are illustrated in Figure 4(c) and (d), respectively. It should be noted that Figure 4(c) presents a porous medium with moderate permeability ( $\mathrm{Da}=0.01$ ) because the similar case is observed in the other porous media. As shown in Figure 4(c), the value of $\overline{\mathrm{Nu}}^{*}$ is reduced with the increasing skew angle from $30^{\circ}$ to $90^{\circ}$; then, it is increased with the further enhancement of $\lambda$ value. In other words, the role of metallic nanoparticles in the heat transfer enhancement rate depends mainly on the skew angle value of parallelogramic porous enclosure. Figure 4(d) shows that the enhancement in rate of heat transfer is increased with Darcy number. Both of Figure 4(c) and (d) demonstrate that heat transfer enhancement of nanofluid due to the addition of metallic nanoparticles is increased by raising the volume fraction of nanoparticles.

\subsection{Effect of magnetic field induction (magnetic Reynolds number, Rem)}

The isopotential contours for cases with various magnetic Reynolds numbers (Rem $=1-100$ ) are represented in Figure 5, where solid and dotted lines are for nanofluid $(\phi=6 \%)$ and pure fluid $(\phi=0)$, respectively. It should be noted that the streamlines and isotherms have insignificant variations with magnetic Reynolds number and, thus, are not presented here for the sake of brevity. The magnetic Reynolds number gives an estimate of the relative effects of advection or induction of a magnetic field by the motion of a conducting fluid to magnetic diffusion. As mentioned before, the magnetic Reynolds number is assumed small enough and magnetic advection is ignored in most of the available studies reported in the literature. However, Figure 5 shows that with the increase of magnetic Reynolds number, the advection effect of external magnetic field is enhanced, whereby isopotential lines circulate through the center of the cavity and follow the movement of the lid. It can be also seen that the distortions caused by magnetic Reynolds number enhancement in non-square parallelogramic enclosures are stronger than those in square enclosures. This fact indicates that the advection effect in non-square parallelogramic porous enclosures is stronger than that in the square porous enclosures and can be attributed to the fluid friction applied against the advection effect of external magnetic field. Square porous enclosures
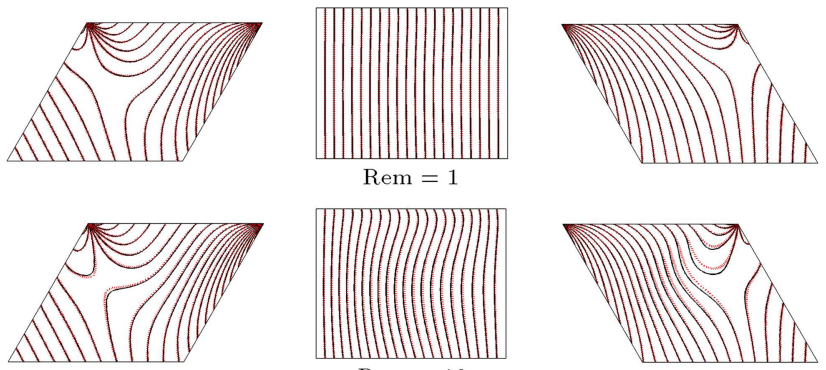

Rem $=10$
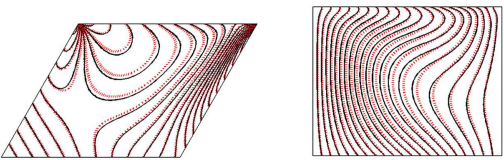

Rem $=50$
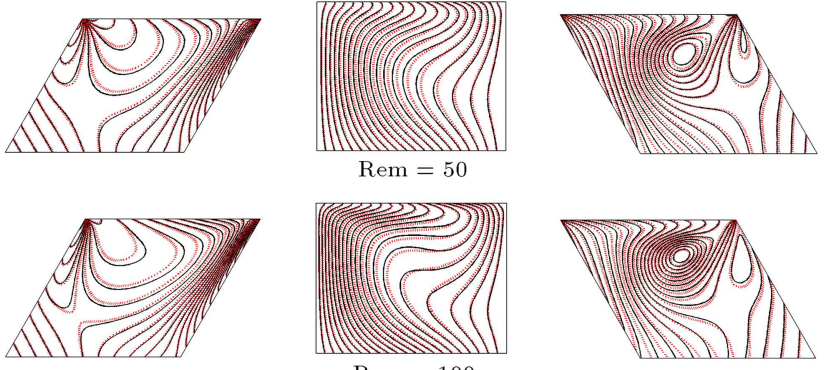

Figure 5. Magnetic isopotentials of MHD forced convection $(\mathrm{Re}=\mathrm{Gr}=100, \mathrm{Ri}=0.01)$ of nanofluid in the parallelogramic porous enclosures with $\lambda=60^{\circ}$ (first column), $\lambda=90^{\circ}$ (second column), and $\lambda=120^{\circ}$ (third column), when $\mathrm{Da}=0.1$ and $\mathrm{Ha}=5$. Dotted lines are for the pure fluid $(\phi=0)$ and solid lines are for the nanofluid $(\phi=6 \%)$.

have a larger effective area than the other parallelogramic porous enclosures; therefore, fluid friction in those enclosures is larger than that in the others. The variations of pure fluid isopotentials (dotted lines) in square porous enclosure are similar to the results of Pekmen and Tezer-Sezgin [14] who investigated numerically MHD convection of pure air in a lid-driven square porous cavity in the presence of magnetic field induction.

Figure 5 also denotes that nanofluid and pure fluid behave similarly with magnetic Reynolds number variation. Nevertheless, it can be seen that the partial difference of nanofluid and pure fluid is increased with increasing Rem value. The variations of $\left|\psi_{\text {Max }}\right|$ values as a function of magnetic Reynolds number for three parallelogramic porous enclosures are depicted in Figure 6(a). It can be seen that the stream function value is reduced slightly with further increasing of Rem in parallelogramic porous cavities. This phenomenon has been also observed before in the study of Kumar and Singh [21], in which the studied the MHD free convection flow past a semi-infinite vertical wall in the presence of an induced magnetic field. The variation of $\overline{\mathrm{Nu}}$ as a function of skew angle for different magnetic Reynolds numbers is illustrated in Figure 6(b). Although a partial reduction is observed in the convection heat transfer, the influence of magnetic Reynolds number on the average Nusselt number seems to be insignificant. 


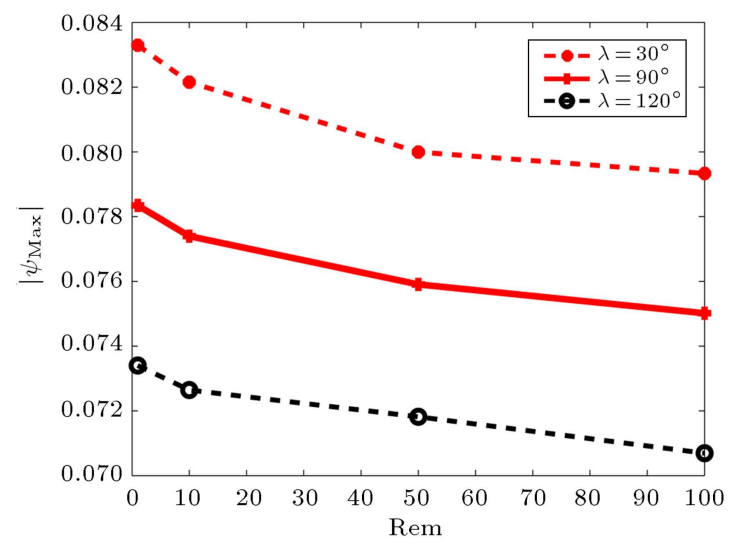

(a)

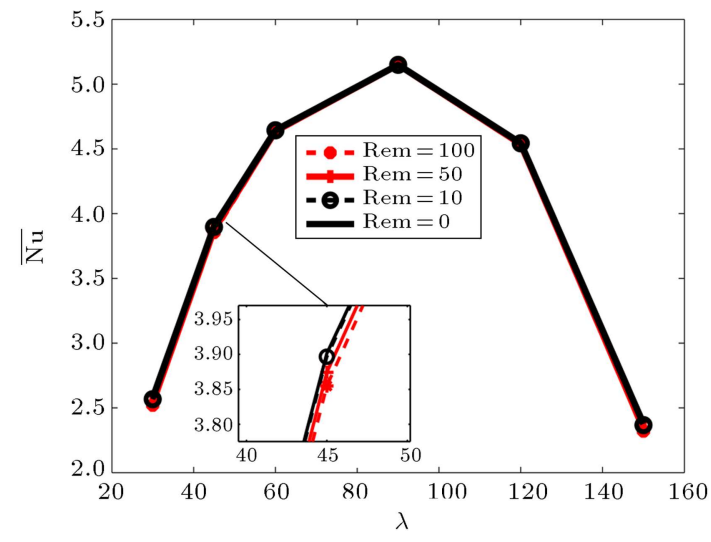

(b)

Figure 6. (a) The variation of the absolute value of stream function of primary vortex as a function of Rem for three parallelogarmic porous enclosures. (b) The variation of the average Nusselt number as a function of skew angle for different magnetic Reynolds numbers, when $\mathrm{Ha}=5, \mathrm{Da}=0.1, \mathrm{Re}=\mathrm{Gr}=100$, and $\mathrm{Ri}=0.01$.

\subsection{Effect of magnetic field strength (Hartman number, $\mathrm{Ha}$ )}

The effect of applied magnetic field is studied here by varying the Hartman number from zero to 100 , while $\mathrm{Da}=0.1$ and $\mathrm{Ri}=1$. Figure 7 represents the streamlines, isotherms, and magnetic isopotentials of nanofluid mixed convection in parallelogramic porous enclosures with various Hartman numbers. As can be observed in Figure 7(a), the parallelogramic porous enclosures are still occupied by a unicellular clockwise vortex. Nanofluid and pure fluid have similar flows, while the nanofluid flow forms smaller vortices with respect to the pure fluid flow. Increasing inertial forces and nanofluid viscosity through the addition of solid volume fraction of nanoparticles is the main reason of this fact, which has been observed before in the study of Nayak et al. [37]. In porous enclosures with $\lambda<90^{\circ}$, the primary vortex expands and moves towards the right side as Hartman number increases. In square porous enclosures, the space occupied by the primary vortex improves with increasing Hartman number. However, in parallelogramic porous enclosures with $\lambda>90^{\circ}$, the primary vortex is compressed and the occupied space is enhanced as Hartman number increases. These phenomena are caused by increasing suppression effect of applied magnetic field against the thermal buoyancy forces and the covering area of skew angle of parallelogramic enclosures. In a mixed convection regime and when natural and forced convections have equivalent importance, applying external magnetic field attenuates thermal buoyancy forces and, thus, causes the enhancement in shear effects. Moving primary vortex towards the right side and increasing occupied space with increasing Hartman number are result of suppression effect of external magnetic field against the thermal buoyancy force exerted from bottom heated wall. Many previous investigators, e.g. Mahmoud et al. [42], and Seth and Sarkar [25], reported the suppression effect of the applied magnetic field against thermal buoyancy forces.

The covering area of the skew angle of parallelogramic enclosure is another factor that affects on the fluid flow pattern when Hartman number increases. In the porous enclosures with $\lambda>90^{\circ}$ which have larger covering area with respect to the others, increasing Hartman number causes a reduction in the size of the primary vortex. The influence of covering area is also observed in Figure 7(b), where isotherm patterns for different Hartman numbers are illustrated. In porous enclosures with $\lambda<90^{\circ}$, which have a smaller covering area with respect to the other enclosures, thermal eddies are formed on the left-hand side of the parallelogramic enclosure due to the competition between thermal buoyancy and shear forces in that region. The thermal eddies move toward the bottom with increasing Hartman number due to the suppression effect of the magnetic field. Moreover, the intensified forced flow forms a concentrated thermal boundary layer in the bottom right corner of enclosure. The same trend is observed in parallelogramic enclosures with $\lambda>90^{\circ}$. In contract, a different behaviour is observed in the square parallelogramic enclosures. It can be seen that the concentration of thermal boundary layers in the near heated wall is reduced and the isotherms become parallel to the horizontal wall with increasing Hartman number, whereby the enclosure becomes a quasi-conductive domain at the highest values of Hartman number. It seems that the effect of applied magnetic field on the isotherm patterns manifests various fashions mainly depending on the skew angle of the parallelogramic porous cavity. The various effects of the external magnetic field on isotherm patterns can be examined as the dual effect of Hartman number on convective heat transfer parallelogramic porous enclosures. A similar dual effect of magnetic field has been also observed before in the 

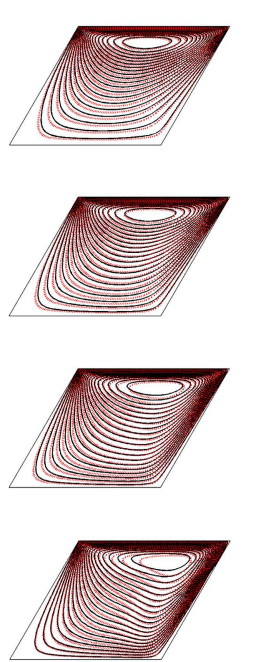

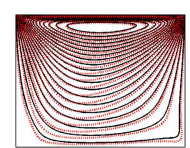

$\mathrm{Ha}=5$

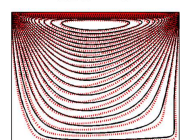

$\mathrm{Ha}=25$

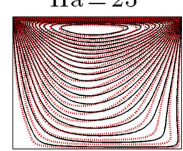

$\mathrm{Ha}=50$

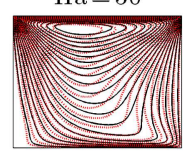

$\mathrm{Ha}=100$

(a)
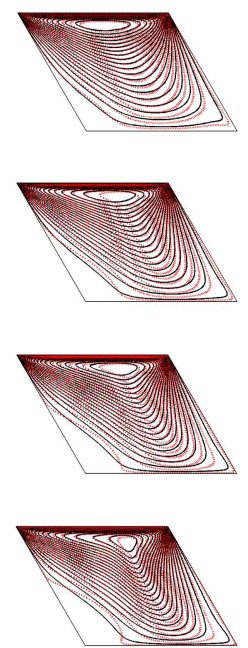
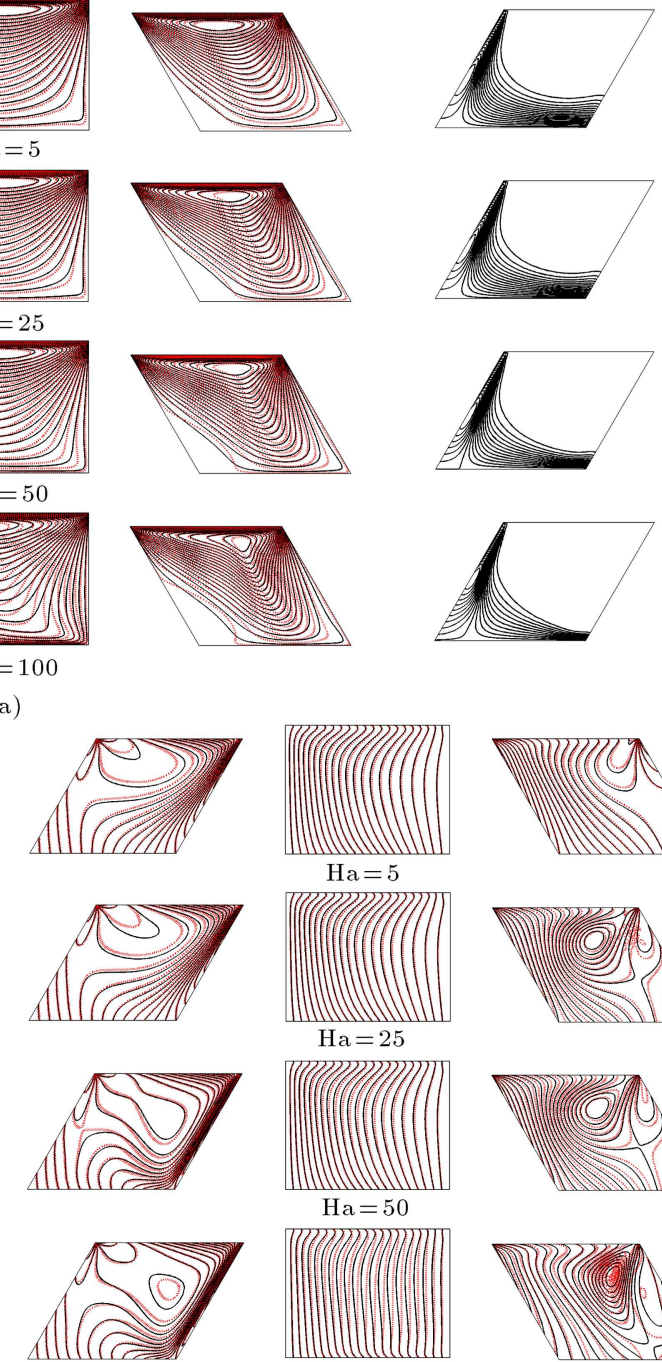

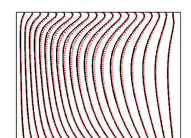

$\mathrm{Ha}=5$

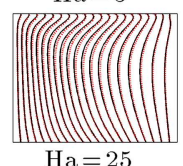

$\mathrm{Ha}=25$

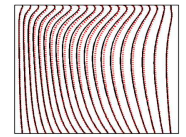

$\mathrm{Ha}=50$

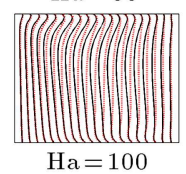

(c)
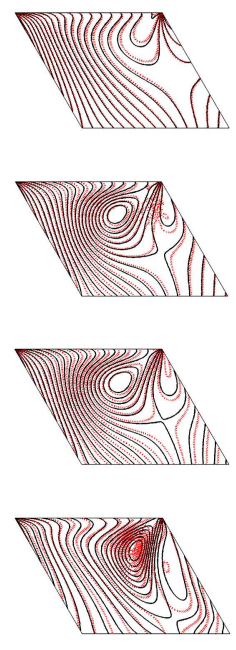

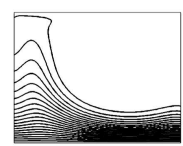

$\mathrm{Ha}=5$

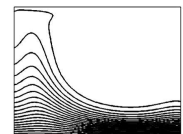

$\mathrm{Ha}=25$

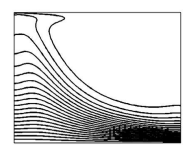

$\mathrm{Ha}=50$

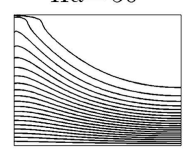

$\mathrm{Ha}=100$

(b)
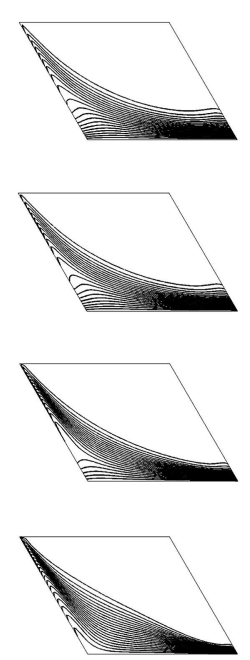

Figure 7. (a) Streamlines, (b) isotherms, and (c) magnetic isopotentials of MHD mixed convection $(\mathrm{Ri}=1)$ of nanofluid in the parallelogramic porous enclosures with $\lambda=60^{\circ}$ (first column), $\lambda=90^{\circ}$ (second column), and $\lambda=120^{\circ}$ (third column), when Rem $=100$, and $\mathrm{Da}=0.1$. Dotted lines are for the pure fluid $(\phi=0)$ and solid lines are for the nanofluid $(\phi=6 \%)$.

study of Mehrez et al. [43]. They studied the effect of an oriented external magnetic field on the heat transfer of nanofluid flow in an open cavity heated from below. Results show that the external magnetic field has a dual effect on convective heat transfer in according to its inclination angle. In particular, they observed that the increase of Hartman number causes the enhancement of convective heat transfer when magnetic field is not applied in the direction of characteristic velocity.

Figure $7(\mathrm{c})$ shows the magnetic isopotential patterns of nanofluid in a parallelogramic porous enclosure for various Hartman numbers. As can be observed in the cases with $\lambda=90^{\circ}$, the intensified magnetic field reduces distortions and makes the magnetic potential lines parallel to each other, representing that the external applied magnetic field tends to reduce the induced magnetic field. The advection effect of the magnetic field by the motion of the conducting pure fluid reduces as the strength of external magnetic field, Hartman number value, increases. This phenomenon has been also observed before by Pekmen and Tezer-Sezgin [14] and Seth and Sarkar [25]. Moreover, it can be seen that nanofluid behaves similarly to the pure fluid. Nevertheless, the presence of nanoparticles increases fluid friction and, thus, reduces the advection effect of the magnetic field. The behaviour of magnetic isopotential contours with Hartman number variation in non-square parallelogramic enclosures deals with the dual effect of external magnetic field, as explained before. As the Hartman number increases, forced flow pushes to penetrate much deeper into the non-square parallelogramic enclosures. In accordance with the value of Rem, the advection distortion through the non-square enclosures increases as the Hartman number improves. 


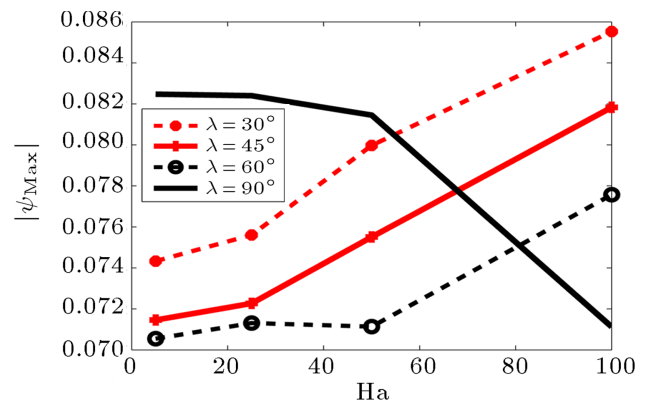

(a)

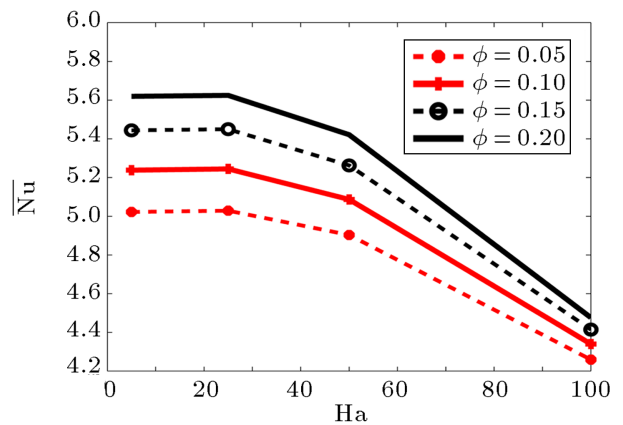

(c)

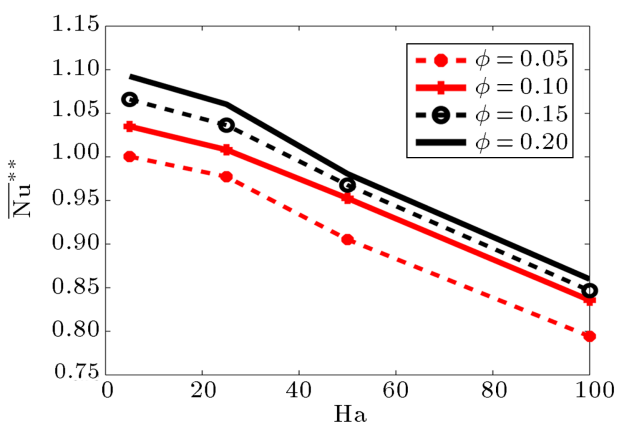

(e)

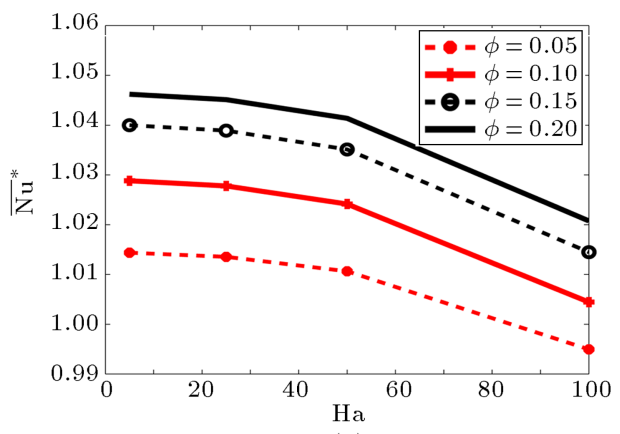

(g)

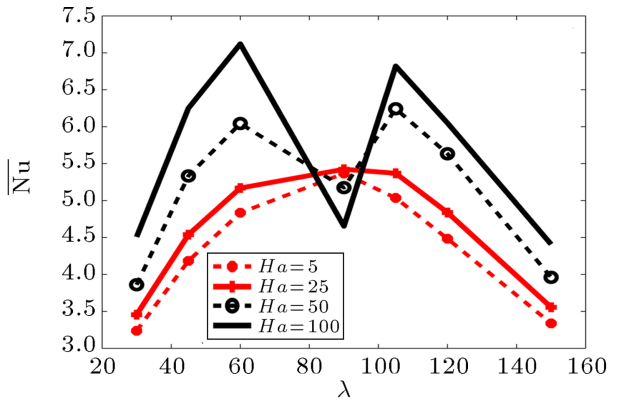

(b)

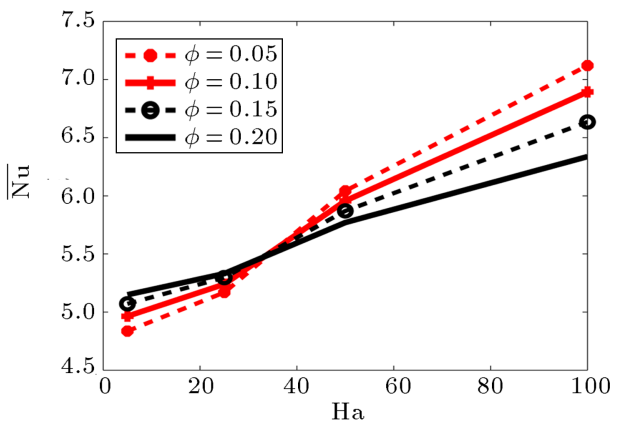

(d)

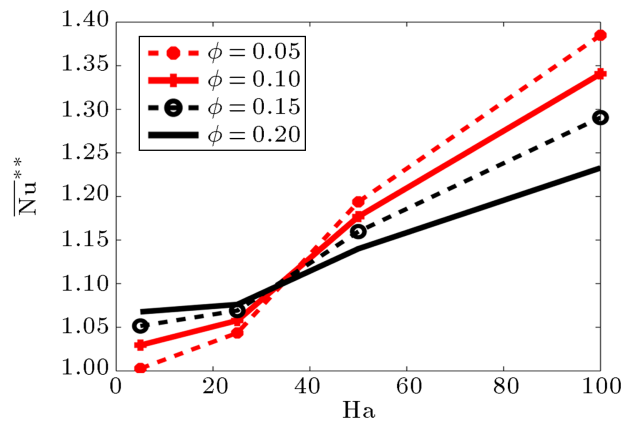

(f)

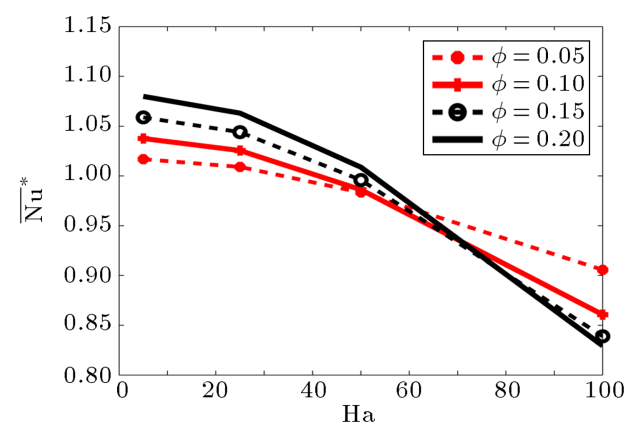

(h)

Figure 8. (a) The variation of the absolute value of stream function of primary vortex as a function of Hartman number. (b) The variation of the average Nusselt number as a function of skew angle for different Hartman numbers. The variation of the average Nusselt number as a function of Hartman number for various solid volume fractions when (c) $\lambda=90^{\circ}$, and (d) $\lambda=60^{\circ}$. The variation of $\overline{\mathrm{Nu}}^{* *}$ as a function of Hartman number for various solid volume fractions when (e) $\lambda=90^{\circ}$ and (f) $\lambda=60^{\circ}$. The variation of $\overline{\mathrm{Nu}}^{*}$ as a function of Hartman number for various solid volume fractions when (g) $\lambda=90^{\circ}$, and $(\mathrm{h}) \lambda=60^{\circ}$. In all figures $\mathrm{Da}=0.1$, Rem $=100$, and $\mathrm{Ri}=1$.

The variations of the absolute value of stream function at the primary vortex as a function of Hartman number are presented in Figure 8(a). The dual effect of Hartman number is also observed here, whereby $\left|\psi_{\text {Max }}\right|$ is decreased and increased with in- creasing Hartman number in square and non-square parallelogramic enclosures, respectively. Increasing Hartman number attenuates thermal buoyancy forces and, thus, reduces $\left|\psi_{\text {Max }}\right|$ values in square enclosures, while it has an aiding effect on forced flow and increases 
$\left|\psi_{\text {Max }}\right|$ in non-square parallelogramic enclosures. To highlight the influence of Hartman number on the heat transfer, the evolution of the average Nusselt number as a function of skew angle for different Hartman numbers is elucidated in Figure $8(\mathrm{~b})$. The average Nusselt number is reduced by increasing Hartman number in the square enclosures, while the opposite is observed in the other parallelogramic enclosures. This fact refers again to the dual effect of Lorenz force in parallelogramic enclosures. The evolution of $\overline{\mathrm{Nu}}$ for parallelogramic enclosures with $\lambda=90^{\circ}$ and $60^{\circ}$ is represented in Figure 8(c) and (d), respectively. It should be noticed that a similar behaviour is observed

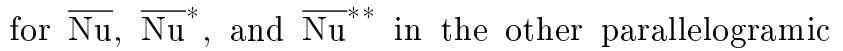
enclosures with $\lambda \neq 90^{\circ}$; therefore, the variations of those parameters are reported here in the cases of $\lambda=60^{\circ}$ for the sake of brevity. When $\lambda=$ $90^{\circ}$ (Figure $8(\mathrm{c})$ ), as the intensity of magnetic field increases, the convection mechanism into the cavity is suppressed and, thus, $\overline{\mathrm{Nu}}$ is reduced. Moreover, heat transfer is improved by increasing solid volume fraction of nanoparticles. This fact attributes to an increase in the thermal conductivity of the nanofluid because of the existence of high electrical conductive nanoparticles in the nanofluid. Figure $8(\mathrm{~d})$ shows that $\overline{\mathrm{Nu}}$ in nonsquare porous enclosures increases as Hartman number increases. When $\lambda \neq 90^{\circ}$, the produced Lorentz force allows the cold fluid to penetrate into the cavity without the recirculation, improving the heat transfer. On the other hand, increasing solid volume fraction, which results in increasing fluid friction, decreases a dominant forced convection mode and, thus, reduces $\overline{\mathrm{Nu}}$ values.

The influence of the magnetic field on $\overline{\mathrm{Nu}}^{* *}$ values in the parallelogramic porous enclosures with $\lambda=90^{\circ}$ and $60^{\circ}$ is displayed in Figure 8(e) and (f), respectively.
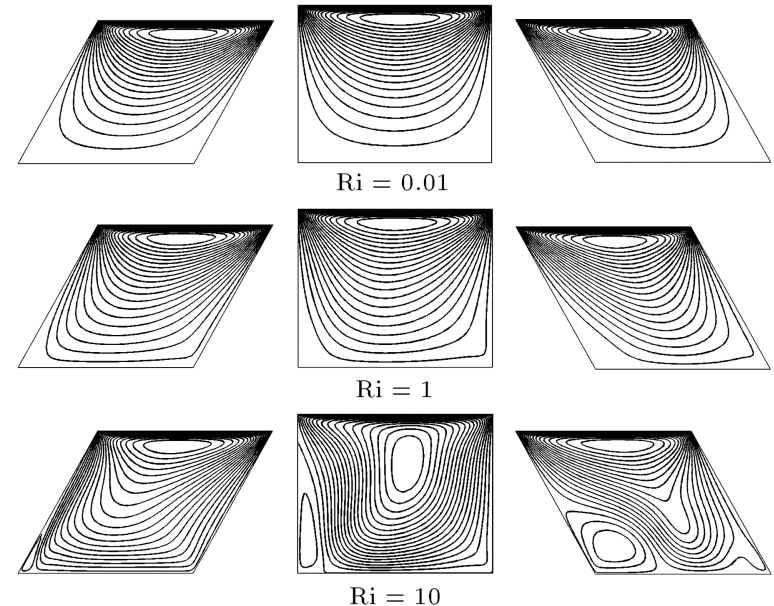

(a)

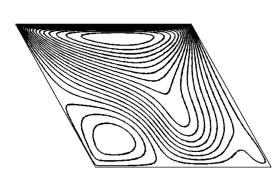

It can be seen that for $\lambda=90^{\circ}, \overline{\mathrm{Nu}}^{* *}$ is decreased by increasing Hartman number, while the opposite is observed for $\lambda=60^{\circ}$. Figure $8(\mathrm{~g})$ and $(\mathrm{h})$ present the variation of $\overline{\mathrm{Nu}}^{*}$ versus $\mathrm{Ha}$ for various solid volume fractions and for two parallelogramic porous enclosures with $\lambda=90^{\circ}$ and $60^{\circ}$, respectively. Results show that, with an increase in Hartman number, only minor enhancement of $\overline{\mathrm{Nu}}^{*}$ is achieved as a result of the presence of nanoparticles. Nevertheless, adding more volume fraction of nanoparticles in the presence of a strong external magnetic field $(\mathrm{Ha}=100)$ and in nonsquare parallelogramic porous enclosures (Figure $8(\mathrm{~h})$ ) increases fluid viscosity/friction and, thus, reduces heat transfer of nanofluid with respect to the base fluid.

\subsection{Effect of buoyancy force intensity} (Richardson number, Ri)

In what follows, the effect of Richardson number on the fluid flow, magnetic potential, and heat transfer characteristics in the presence of magnetic field induction (Rem $=100$ ) will be discussed. In fact, the Richardson number value identifies the importance of natural convection relative to the forced convection in the thermal convection problems. The streamlines and isopotential patterns of MHD mixed convection of nanofluid in three parallelogramic porous enclosures for three convection regimes, i.e., forced $(\mathrm{Ri}=0.01)$, mixed $(\mathrm{Ri}=1)$, and natural convections $(\mathrm{Ri}=10)$, are depicted in Figure 9(a) and (b), respectively. It is worthwhile to note that Richardson number varies here by increasing Grashof number, when Reynolds number is kept constant $(\operatorname{Re}=100)$. Figure 9(a) elucidates that the primary vortex occupying the whole cavity moves downwards and also expands vertically as Richardson number improves. The increasing convective current of heat due to the enhancement of
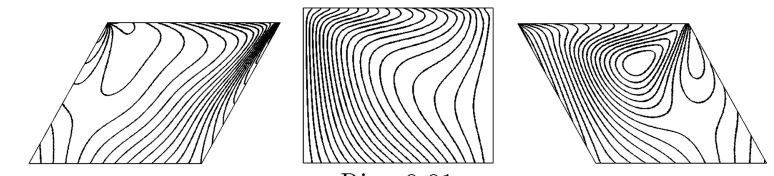
$\mathrm{Ri}=0.01$
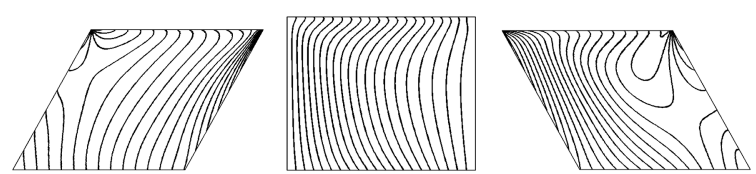

$\mathrm{Ri}=1$
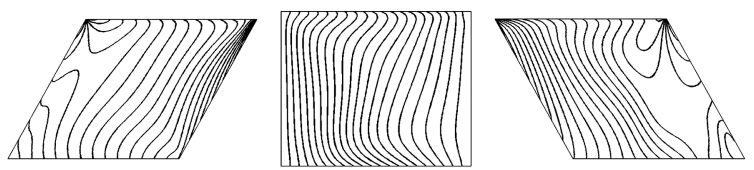

$\mathrm{Ri}=10$

(b)

Figure 9. (a) Streamlines and (b) magnetic isopotentials of MHD convection of nanofluid in the parallelogramic porous enclosures with $\lambda=60^{\circ}$ (first column), $\lambda=90^{\circ}$ (second column), and $\lambda=120^{\circ}$ (third column), when Rem $=100$, $\mathrm{Da}=0.1$, and $\mathrm{Ha}=5$. 
Richardson number can be recognized as the main reason of this phenomenon. It can also be seen that the secondary vortices are formed in the left bottom corner of enclosure when Richardson number has its highest value $(\mathrm{Ri}=10)$. Meanwhile, the size of secondary vortices improves as the skew angle of parallelogramic enclosure increase. This may be due to the covering area of skew angle at that region, enhancing as skew angle increases. With increasing Richardson number, the importance of thermal buoyancy forces imposed by bottom heated wall increases. The competition between the buoyancy and shear forces forms secondary vortices in the left bottom corner of enclosure. Increasing covering area, due to increasing skew angle of parallelogramic enclosure, highlights the importance of thermal buoyancy forces and, thus, forms larger secondary vortices.

The influence of Richardson number on isopotential contours is shown in Figure 9(b). As mentioned before, the advection effect of the magnetic field on the electrical conductive medium becomes important when magnetic Reynolds number is a large value (Rem $\gg$ 1). It can be seen that the isopotential contours are affected greatly by lid's movement when flow is dominated by forced convection. However, isopotential

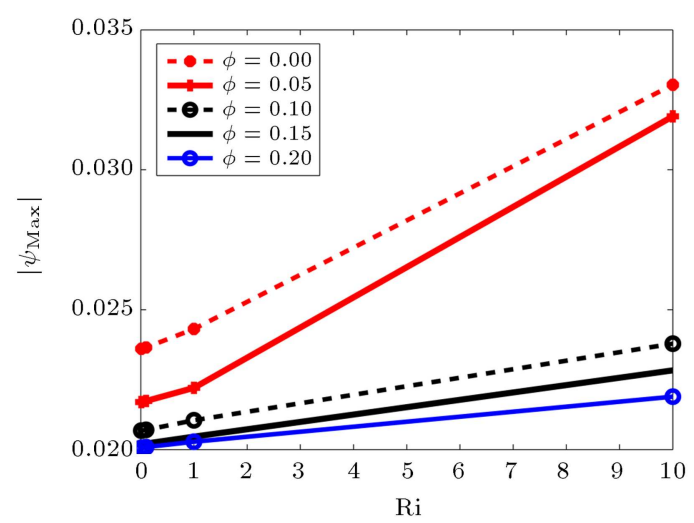

(a)

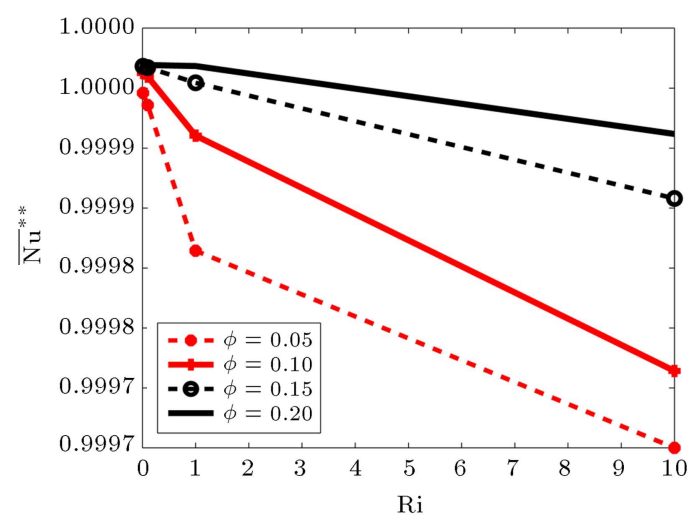

(c) counters are affected greatly by shear forces in nonsquare parallelogramic enclosures because the effective area and fluid friction in those enclosures are smaller than those in square enclosures. The advection effect of magnetic field is attenuated by increasing Richardson number. Nevertheless, the influence of shear forces near the moving lid and thermal buoyancy forces in the vicinity of the bottom heated wall on isopotential counters is well observed in cases with the largest Richardson number.

The variations of absolute value of stream function of primary vortex and average Nusselt number as a function of Richardson number are shown in Figure 10(a) and (b), respectively. It should be noted that the variations are just presented here in a square porous enclosure because a similar case is observed in the other enclosures. It can be seen that both of $\left|\psi_{\text {Max }}\right|$ and $\overline{\mathrm{Nu}}$ values are enhanced with further increasing of Richardson number. This fact can be attributed to an increase in thermal buoyancy forces because of the Richardson number augmentation. The similar was observed before in the study of Mahmoud et al. [42]. However, $\left|\psi_{\text {Max }}\right|$ values are reduced with the further enhancement of solid volume fraction. This fact is due to the increase of viscosity and fluid friction because of

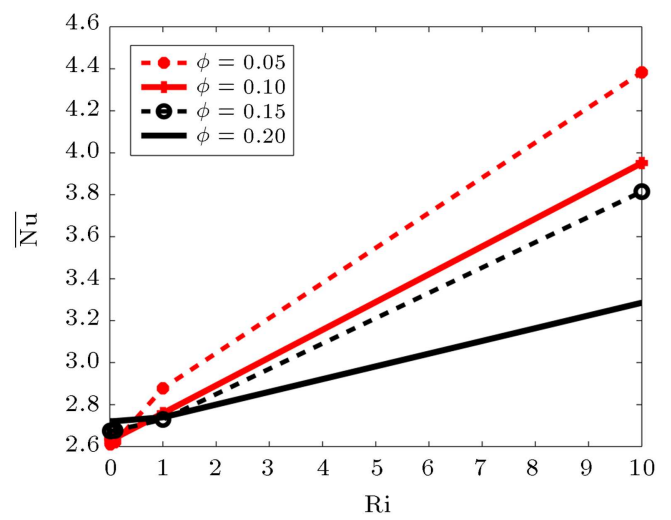

(b)

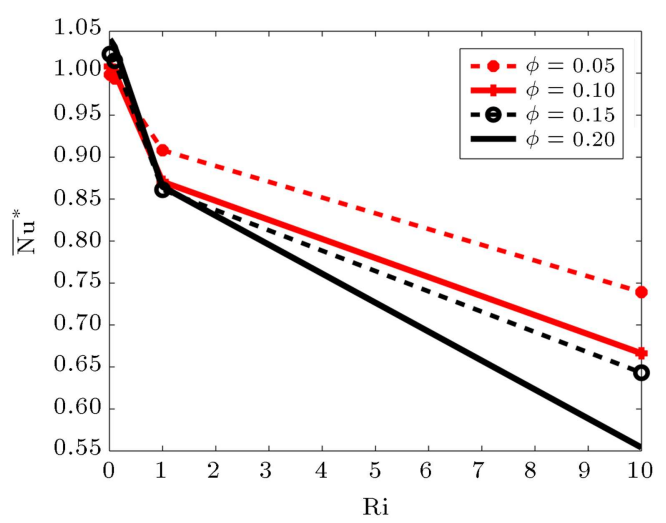

(d)

Figure 10. The variation of (a) absolute value of stream function of primary vortex, (b) average Nusselt number, (c) $\overline{\mathrm{Nu}}^{* *}$, and (d) $\overline{\mathrm{Nu}}^{*}$ in the cases of $\lambda=90^{\circ}$ as a function of Richardson number for various solid volume fractions, when $\mathrm{Da}=0.1$, Rem $=100, \mathrm{Re}=100$, and $\mathrm{Ha}=5$. 
nanoparticles addition. This scenario is not repeated completely in the variation of average Nusselt number. As can be observed in Figure 10(b), $\overline{\mathrm{Nu}}$ decreases as $\phi$ improves in the cases of large and moderate Richardson numbers, while the opposite is observed in the cases of the smallest Richardson number $(\mathrm{Ri}=$ $0.01)$.

In the porous media and in the case of an increase in Richardson number, the enhancement of $\phi$ allows for an increase in fluid friction and local viscosity, while, at the same time, it does not lead to temperature differences. Hence, an increase in $\phi$ leads to conserving the main convective cell with upward flows along the bottom heated wall and downward flows close to the top cold wall. Hence, the average Nusselt number is a decreasing function of solid volume fraction of nanoparticles when $\mathrm{Ri} \geq 1$. The variations of $\overline{\mathrm{Nu}}^{* *}$ and $\overline{\mathrm{Nu}}^{*}$ as functions of Richardson number for various solid volume fractions of nanoparticles are illustrated in Figure 10(c) and (d), respectively. It can be seen that the influence of applying external magnetic field and adding nanoparticles to the base fluid on the heat transfer variations of the nanofluid is reduced with Richardson number, whereby both of $\overline{\mathrm{Nu}}^{* *}$ and $\overline{\mathrm{Nu}}^{*}$ are decreasing functions of Ri. Moreover, the cases with larger $\phi$ values have smaller $\overline{\mathrm{Nu}}^{* *}$ values. In other words, adding metallic nanoparticles in the presence of magnetic field induction reduces greatly the convective heat transfer within the enclosure. On the other hand, adding nanoparticles in the porous media enhances viscosity and fluid friction, whereby it attenuates the convective current of energy across the enclosure. Hence, the cases of smaller solid volume fraction have larger $\overline{\mathrm{Nu}}^{*}$ values.

\section{Conclusion}

This study investigated numerically the flow field and heat transfer of mixed convection flow in a differentially heated lid-driven parallelogramic porous enclosure saturated with a water-based nanofluid and subjected to an external applied magnetic field. The induced magnetic field was also taken into account and was considered in terms of magnetic potential. The flow, thermal and magnetic potential fields were illustrated by the streamlines, isotherm, and isopotential contours, respectively. Particulate efforts were focused on the effects of the skew angle, solid volume fraction parameter of nanoparticles, Darcy, magnetic Reynolds, Hartman, and Richardson numbers on the flow, thermal, magnetic potential fields as well as the average Nusselt number. The main findings of this study can be highlighted as follows:

1. It was found that the reduction of porous permeability caused a slow-down in the nanofluid motion within the porous media and made a reduction in the heat transfer across the parallelogramic porous enclosure;

2. The effect of magnetic Reynolds number on the heat transfer and fluid flow was found to be insignificant. Nevertheless, some partial effects were detected in the non-square parallelogramic porous enclosures;

3. Fluid intensity and heat transfer in parallelogramic porous enclosures under the small external magnetic fields were found sensitive enough to the skew angle and effective area. The absolute value of stream function at primary vortex, which deals with fluid intensity, was minimum for square parallelogramic porous enclosures, while average Nusselt number had its maximum value for those porous enclosures;

4. The influence of magnetic field on both of heat transfer and fluid flow depends mainly on the skew angle of parallelogramic porous enclosure, whereby a dual effect of Lorenz force was observed during data results. For square enclosures, the intensity of recirculation into the cavity was attenuated and the thermal boundary layers in the vicinity of the bottom heated wall became less concentrated by increasing Hartman number. However, when $\lambda \neq$ $90^{\circ}$, the augmentation of Hartman number led to the suppression of the recirculation and helped force convection dominancy;

5. The heat transfer enhancement rate because of existing metallic nanoparticles also depends mainly on skew angle value of parallelogramic porous enclosure;

6. At a fixed skew angle, the variation in rate of heat transfer by adding nanoparticles to the base fluid was reduced with the enhancement of Richardson number. The enhancement rate also decreased with the increase of solid volume fraction.

\section{Nomenclature}

$\begin{array}{ll}\overline{\mathrm{Nu}} & \text { Average Nusselt number } \\ B_{0} & \text { Magnetic field intensity }\left(\mathrm{Wbm}^{-2}\right) \\ C & \text { Forchheimer coefficient } \\ \mathrm{Da} & \text { Darcy number }\left(\mathrm{KH}^{-2}\right) \\ g & \text { Acceleration due to gravity }\left(\mathrm{ms}^{-2}\right) \\ \mathrm{Gr} & \text { Grashof number } \\ K & \text { Permeability of porous medium }\left(\mathrm{m}^{2}\right) \\ L & \text { Enclosure length }(\mathrm{m}) \\ \mathrm{Nu} & \text { Local Nusselt number } \\ P & \text { Non-dimensional } \\ p & \text { Fluid pressure }\left(\mathrm{Nm}{ }^{-2}\right)\end{array}$




\begin{tabular}{|c|c|}
\hline $\operatorname{Pr}$ & Prandtl number \\
\hline$R_{k}$ & $\begin{array}{l}\text { Thermal conductivity ratio porous- } \\
\text { nano }\end{array}$ \\
\hline$R_{\mu}$ & Viscosity ratio porous-nano \\
\hline $\operatorname{Re}$ & Reynolds number \\
\hline $\mathrm{Ri}$ & Richardson number \\
\hline$T$ & Fluid temperature (K) \\
\hline$U, V$ & Non-dimensional velocity components \\
\hline$u, v$ & Velocity components $\left(\mathrm{ms}^{-1}\right)$ \\
\hline$U_{0}$ & Absolute lid velocity \\
\hline$X, Y$ & Non-dimensional Cartesian coordinate \\
\hline$x, y$ & Cartesian coordinate $(\mathrm{m})$ \\
\hline \multicolumn{2}{|c|}{ Greek } \\
\hline$\alpha$ & Thermal diffusivity $\left(\mathrm{m}^{2} \mathrm{~s}\right)$ \\
\hline$\beta$ & Thermal expansion coefficient $\left(\mathrm{K}^{-1}\right)$ \\
\hline$\eta$ & Transformed coordinate in $y$-direction \\
\hline$\mu$ & Dynamic viscosity $\left(\mathrm{kgm}^{-1} \mathrm{~s}^{-1}\right)$ \\
\hline$\nu$ & Kinematic viscosity $\left(\mathrm{m}^{2} \mathrm{~s}^{-1}\right)$ \\
\hline$\phi$ & Volume fraction of the nanoparticles \\
\hline$\rho$ & Density $\left(\mathrm{kgm}^{-3}\right)$ \\
\hline$\sigma$ & Electrical conductivity $\left(\mathrm{Sm}^{-1}\right)$ \\
\hline$\Theta$ & Dimensionless temperature \\
\hline$\varepsilon$ & Porosity \\
\hline$\zeta$ & Transformed coordinate in $\mathrm{x}$-direction \\
\hline
\end{tabular}

\section{Superscript}

$h \quad$ High temperature

$l \quad$ Low temperature

$s \quad$ Solid particles

\section{References}

1. Nield, D.A. and Bejan, A., Convection in Porous Media, 4th Edition, New York, Springer (2013).

2. Sahoo, S.N., Panda, J.P., and Dash, G.C. "The MHD mixed convection stagnation point flow and heat transfer in a porous medium", In Proceedings of National Academy Sciences, India, Section A Physical Sciences, 83, pp. 371-381 (2013).

3. Shermet, M.A. and Pop, I. "Thermo-Bi convection in a square porous cavity filled by oxytactic microorganisms", Trans. Porous Media, 103, pp. 191-205 (2014).

4. Ghalambaz, M., Moattar, F., Shermet, M.A., and Pop, I. "Triple-diffusive natural convection in a square porous cavity", Trans. Porous Media, 111, pp. 59-79 (2016).

5. Mahmoud, A., Mejri, I., Abbasi, M.A., and Omri, A. "Analysis of MHD natural convection in a nanofluidfilled open cavity with non-uniform boundary condition in the presence of uniform heat generation/absorption", Powder Technol., 269, pp. 275-289 (2015).
6. Sheikholeslami, M., Hatami, M., and Ganji, D.D. "Analytical investigation of MHD nanofluid flow in a semi-porous channel", Powder Technol., 246, pp. 327336 (2013).

7. Servati, A.A., Javaherdeh, K., and Ashorynejad, H.R. "Magnetic field effects on force convection flow of a nanofluid in a channel partially filled with porous media using lattice Boltzmann method", Adv. Powder Technol., 25, pp. 666-675 (2014).

8. Choi, S.U.S. "Enhancing thermal conductivity of fluid with nanoparticles", ASME Fluid Eng. Div., 23, pp. 99-105 (1995).

9. Fersadou, I., Kahalerras, H., and El Ganaoui, M. "MHD mixed convection and entropy generation of a nanofluid in a vertical porous channel", Computers and Fluids, 121, pp. 164-179 (2015).

10. Ziaei-Rad, M., Saeedan, M., and Afshari, E. "Simulation and prediction of MHD dissipative nanofluid flow on a permeable stretching surface using artificial neural network", Appl. Thermal Eng., 99, pp. 373-382 (2016).

11. Ziaei-Rad, M., Kasaeipoor, A., Rashidi, M.M., and Lorenzini, G. "A similarity solution for mixedconvection boundary layer nanofluid flow permeable surface", J. Thermal Sci. Eng. Appl., 9, p. 021015 (2017).

12. Zeeshan, A., Ellahi, R., and Hasan, M. "Magnetohydrodynamic flow of water/ethylene glycol based nanofluids with natural convection through a porous medium", The European Physical Journal Plus, 129, pp. 261-271 (2014).

13. Murthy, P.V.S.N., Reddy C., Chamkha, A.J., and Rashad. A.M. "Magnetic effect on thermally stratified nanofluid saturated non-Darcy porous medium under convective boundary condition", Int. Comm. Heat Mass Transfer, 47, pp. 41-48 (2013).

14. Pekmen, B. and Tezer-Sezgin, M. "DRBEM solution of free convection in porous enclosure under the effect of a magnetic field", Int. J. Heat Mass Transfer, 56, pp. $454-468$ (2013).

15. Shermet, M.A., Grosan, T., and Pop, I. "Free convection in a square cavity filled with a porous medium saturated by nanofluid using Tiwari and Das nanofluid model", Trans. Porous Media, 106, pp. 595-610 (2015).

16. Mabood, F., Shateyi, S., Rashidi, M.M., Momoniat, E., and Freidoonimehr, N. "MHD stagnation point flow heat and mass transfer of nanofluids in porous medium with radiation, viscous dissipation and chemical reaction", Adv. Powder Technol., 27, pp. 742-749 (2016).

17. Sarris, I.E., Zikos, G.K., Grecos, A.P., and Vlachos, N.S. "On the limits of validity of the low magnetic Reynolds number approximation in MHD naturalconvection heat transfer", Num. Heat Transfer, Part B: Fundamentals: An International Journal of Computation and Methodology, 50, pp. 157-180 (2006).

18. Baïri, A., Zarco-Pernia, E., and Garćia de Maria, J.M. "A review of natural convection in enclosures for 
engineering applications: The particular case of the parallelogrammic diode cavity", Appl. Ther. Eng., 63, pp. 304-322 (2014).

19. Singh, R.K., Singh, A.K., Sacheti, N.C., and Chandran, P. "On hydromagnetic free convection in the presence of induced magnetic field", Heat Mass Transfer, 46, pp. 523-529 (2010).

20. Costa, V.A.F., Sousa, A.C.M., and Vasseur, P. "Natural convection in square enclosures filled with fluidsaturated porous media under the influence of the magnetic field induced by two parallel vertical electric currents", Int. J. Heat Mass Transfer, 55, pp. 73217329 (2012).

21. Kumar, A. and Singh, A.K. "Unsteady MHD free convective flow past a semi-infinite vertical wall with induced magnetic field", Appl. Math. Comput., 222, pp. 462-471 (2013).

22. Akbar, N.S., Raza, M., and Ellahi, R. "Interaction of nanoparticles for the peristaltic flow in an asymmetric channel with the induced magnetic field", The European Physical Journal Plus, 129, pp. 155-167 (2014).

23. Akbar, N.S., Raza, M., and Ellahi, R. "Influence of heat generation and heat flux on peristaltic flow with interacting nanoparticles", The European Physical Journal Plus, 129, pp. 185-190 (2014).

24. Pekmen, B. and Tezer-Sezgin, M. "MHD flow and heat transfer in a lid driven porous enclosure", Computers and Fluids, 89, pp. 191-199 (2014).

25. Seth, G.S. and Sarkar, S. "Hydromagnetic natural convection flow with induced magnetic field and nth order chemical reaction of a heat absorbing fluid past an impulsively moving vertical plate with ramped temperature", Bul. Chem. Commun., 47, pp. 66-79 (2015).

26. Kumar, V.A. "Numerical study of effect of induced magnetic field on transient natural convection over a vertical cone", Alexandria Eng. J., 55 pp. 1211-1223 (2016).

27. Demirdzic, I., Lilek, Z., and Peric, M. "Fluid flow and heat transfer test problems for non-orthogonal grids: bench-mark solutions", Int. J. Num. Meth. Fluids, 15, pp. 329-354 (1992).

28. Roychowdhury, D.G., Das, S.K., and Sundarsrajan, T. "An efficient solution method for incompressible Navier-Stokes equations using non-orthogonal collected grid", Int. J. Num. Methods Eng., 45, pp. 741763 (1999).

29. Wang, Y. and Komori, S. "On the improvement of the SIMPLE-Like method for flows with complex geometry", Heat Mass Transfer, 36, pp. 71-78 (2000).

30. Qu, Z.G., Tao, W.Q., and Hw, Y.L. "An improved numerical scheme for the SIMPLER method on nonorthogonal curvilinear coordinates: SIMPLER", Num. Heat Transfer Part B: Fundamentals, 51, pp. 43-66 (2007).

31. Costa, V.A.F. "Double-diffusive natural convection in parallelogrammic enclosure", Int. J. Heat Mass Transfer, 47, pp. 2913-2926 (2006).
32. Al-Farhany, K. and Turan, A. "Numerical study of double diffusive natural convective heat and mass transfer in an inclined rectangular cavity filled with porous medium", Int. Commun. Heat Mass Transfer, 39, pp. 174-181 (2012).

33. Chamkha, A.J., Hussain, S.H., and Ali, F.H. "Conduction-combined forced and natural convection in a lid-driven parallelogram-shaped enclosure divided by a solid partition", Progress in Comput. Fluid Dyn., 12, pp. 309-321 (2012).

34. Jagadeesha, R.D., Prasanna, B.M.R., and Sarkar, M. "Double diffusive convection in an inclined parallelogrammic porous enclosure", Procedia Engineering, 127, pp. 1346-1353 (2015).

35. Le Dez, V. and Sadat, H. "Radiation heat transfer in a parallelogram shaped cavity", Int. Commun. Heat Mass Transfer, 68, pp. 137-149 (2015).

36. Hussein, S.H. and Hussain, A.K. "Natural convection heat transfer enhancement in a differentially heated parallegramic enclosure filled with copperwater nanofluid", ASME J. Heat Transfer, 136, pp. 82502-82508 (2014).

37. Nayak, R.K., Bhattacharyya, S., and Pop, I. "Numerical study on mixed convection and entropy generation of $C u$-water nanofluid in a differentially heated skewed enclosure", Int. J. Heat Mass Transfer, 85, pp. 620-634 (2015).

38. Ghaffarpasand, O. "Conjugate effect of Joule heating and unsteady MHD natural convection in a differentially heated skewed porous cavity saturated by $\mathrm{Cu}$ water nanofluid", J. Appl. Fluid Mech., 9, pp. 28232836 (2016).

39. Tiwari, R.K. and Das. M.K. "Heat transfer augmentation in a two-sided lid-driven differentially heated square cavity utilizing nanofluids", Int. J. Heat Mass Transfer, 50, pp. 2002-2018 (2007).

40. Hamilton, R.I. and Crosser, O.K. "Thermal conductivity of heterogeneous two-component systems", Ind. Eng. Chem. Fund., 1, pp. 187-191 (1962).

41. Abu-Nada, E. and Chamkha, A.J. "Mixed convection flow of a nanofluid in a lid-driven cavity with wavy wall", Int. Commun. Heat Mass Transfer, 57, pp. 3647 (2014).

42. Mahmoud, A., Mejri, I., Abbasi, M.A., and Omri, A. "Analysis of MHD natural convection in a nanofluidfilled open cavity with non-uniform boundary condition in the presence of uniform heat generation/absorption", Powder Technol., 269, pp. 275-289 (2015).

43. Mehrez, Z., Cafsi, A.E., Belghith, A., Quéré, P.Le. "MHD effects and entropy generation of nanofluid flow in an open cavity", J. Mag. Mag. Mat., 374, pp. 214224 (2015).

\section{Biographies}

Omid Ghaffarpasand received his $\mathrm{PhD}$ from the Department of Physics, University of Isfahan, Iran, in 
2012. Currently, he is a full-time Faculty Member of the Department of Physics and also the Head of research group of air pollution and solid waste matter, environmental research institute, University of Isfahan, Iran. His current research fields include Computational Fluid Dynamics (CFD), aerosol technology, aerosol propagation and deposition mechanisms, aerosol sizing techniques, heat and mass transfer and magnetohydrodynamic convection in various regimes.

Dariush Fazeli received his master degree in Physics from the Department of Physics, University of Isfahan, Iran, in 2017. He is now a Physics Teacher in high schools of Shush, Ahvaz, Iran. 